\title{
TNF-driven adaptive response mediates resistance to EGFR inhibition in lung cancer
}

\author{
Ke Gong, ${ }^{1}$ Gao Guo,, David E. Gerber, ${ }^{2,3}$ Boning Gao, ${ }^{4,5}$ Michael Peyton, ${ }^{4,5}$ Chun Huang, ${ }^{4,5}$ John D. Minna, ,4,5 Kimmo J. Hatanpaa, ${ }^{6}$ \\ Kemp Kernstine, ${ }^{7}$ Ling Cai, ${ }^{8}$ Yang Xie, ${ }^{8}$ Hong Zhu, ${ }^{3,9}$ Farjana J. Fattah, ${ }^{3}$ Shanrong Zhang, ${ }^{10}$ Masaya Takahashi, ${ }^{10}$ \\ Bipasha Mukherjee, ${ }^{11}$ Sandeep Burma, ${ }^{11}$ Jonathan Dowell, ${ }^{2,12}$ Kathryn Dao, ${ }^{13}$ Vassiliki A. Papadimitrakopoulou, ${ }^{14}$ \\ Victor Olivas, ${ }^{15}$ Trever G. Bivona, ${ }^{15}$ Dawen Zhao, ${ }^{16}$ and Amyn A. Habib ${ }^{1,3,12}$ \\ 'Department of Neurology and Neurotherapeutics, ${ }^{2}$ Internal Medicine, Division of Hematology-Oncology, ${ }^{3}$ Harold C. Simmons Comprehensive Cancer Center, ${ }^{4}$ Department of Pharmacology, \\ ${ }^{5}$ Hamon Center for Therapeutic Oncology Research, ${ }^{6}$ Department of Pathology, ${ }^{7}$ Department of Cardiovascular and Thoracic Surgery, ${ }^{8}$ Quantitative Biomedical Research Center, ${ }^{9}$ Department of Clinical \\ Sciences, ${ }^{10}$ Department of Radiology, and ${ }^{11}$ Department of Radiation Oncology, University of Texas Southwestern Medical Center, Dallas, Texas, USA. ${ }^{12}$ VA North Texas Health Care System, Dallas, Texas, USA. \\ ${ }^{13}$ Baylor Research Institute, Dallas, Texas, USA. ${ }^{14}$ Department of Thoracic Head and Neck Medical Oncology, MD Anderson Cancer Center, Houston, Texas, USA. ${ }^{15}$ Department of Medicine, UCSF, \\ San Francisco, California, USA. ${ }^{16}$ Departments of Biomedical Engineering and Cancer Biology, Wake Forest School of Medicine, Winston-Salem, North Carolina, USA.
}

\begin{abstract}
Although aberrant EGFR signaling is widespread in cancer, EGFR inhibition is effective only in a subset of non-small cell lung cancer (NSCLC) with EGFR activating mutations. A majority of NSCLCs express EGFR wild type (EGFRwt) and do not respond to ECFR inhibition. TNF is a major mediator of inflammation-induced cancer. We find that a rapid increase in TNF level is a universal adaptive response to EGFR inhibition in NSCLC, regardless of EGFR status. EGFR signaling actively suppresses TNF mRNA levels by inducing expression of miR-21, resulting in decreased TNF mRNA stability. Conversely, EGFR inhibition results in loss of miR-21 and increased TNF mRNA stability. In addition, TNF-induced NF- $\kappa B$ activation leads to increased TNF transcription in a feed-forward loop. Inhibition of TNF signaling renders EGFRwt-expressing NSCLC cell lines and an EGFRwt patient-derived xenograft (PDX) model highly sensitive to EGFR inhibition. In ECFR-mutant oncogene-addicted cells, blocking TNF enhances the effectiveness of EGFR inhibition. EGFR plus TNF inhibition is also effective in NSCLC with acquired resistance to EGFR inhibition. We suggest concomitant EGFR and TNF inhibition as a potentially new treatment approach that could be beneficial for a majority of lung cancer patients.
\end{abstract}

\section{Introduction}

Oncogene addiction has been described primarily in cancers that express oncogenes rendered constitutively active by mutation (1, 2). Constitutive activation results in a continuous and unattenuated signaling, resulting in a widespread activation of intracellular pathways and a reliance of the cell on such pathways for survival (3). A subset of non-small cell lung cancers (NSCLCs) harbor epidermal growth factor receptor (EGFR) activating mutations that render the receptor constitutively active and oncogene addicted (4-6). Lung cancers with activating EGFR mutations exhibit a dramatic initial clinical response to treatment with EGFR tyrosine kinase inhibitors (TKIs) (7), but this is followed by the inevitable development of secondary resistance (8). Major TKI resistance mechanisms include other EGFR mutations such as the T790M mutation that prevent TKI enzyme interaction (9) and activation of other receptor tyrosine kinases such as MET or Axl, providing a signaling bypass to EGFR TKI-mediated inhibition (10-12). Rapid feedback loops with activation of STAT3 may also contribute to EGFR TKI resistance in lung cancer cells with EGFR activating

Authorship note: KG and GG contributed equally to this work. Conflict of interest: The authors have declared that no conflict of interest exists. Submitted: July 7, 2017; Accepted: March 16, 2018.

Reference information: / Clin Invest. 2018;128(6):2500-2518.

https://doi.org/10.1172/JCI96148. mutations $(13,14)$. Multiple additional mechanisms and distinct evolutionary pathways may lead to secondary resistance to EGFR inhibition in lung cancer $(8,15-17)$.

The most common type of EGFR expressed in lung cancer is EGFR wild type (EGFRwt). EGFRwt-expressing tumor cells are not oncogene addicted and are usually resistant to EGFR inhibition. EGFR activating mutations result in constitutive signaling and have been shown to be transforming (18). Compared with EGFRwt, EGFR activating mutations lead to activation of extensive signaling networks that, in turn, lead to dependence of tumor cells on continuous EGFR signaling for survival (3). This is likely the reason that EGFR inhibition is effective in EGFR-mutant NSCLC patients with EGFR activating mutations despite the well-documented generation of early adaptive survival responses such as STAT3 or NF-кB in EGFR-mutant cells $(14,19)$. Increased affinity of mutant EGFR for TKIs has also been reported $(20,21)$.

MicroRNAs are small noncoding RNAs that target coding RNAs, regulate the translation and degradation of mRNAs, and may play an important role in cancer $(22,23)$. Expression levels of microRNAs are altered in various types of cancer, including lung cancer (24). EGFR activity can regulate microRNA levels in lung cancer. The expression of 5 microRNAs (hsa-miR-155, hsa-miR-173p, hsa-let-7a-2, hsa-miR-145, and hsa-miR-21) was altered in lung cancer from smokers compared with uninvolved lung tissue (25), and there is evidence that EGFR activity upregulates the expres- 
sion of miR-21 while inhibition of EGFR activity downregulates miR-21. Both EGFRwt and mutant activity may regulate miR-21 in lung cancer, although EGFR activating mutants appear to have a stronger effect $(25,26)$.

In this study, we demonstrate that a rapid increase in TNF levels is a universal response to inhibition of EGFR signaling in lung cancer cells, regardless of whether EGFR is mutant or wild type. EGFR regulates TNF mRNA stability via miR-21 and by activation of NF- $\kappa \mathrm{B}$ that leads to a further increase in TNF transcription, generating a feed-forward loop. The biological effect of this TNFdriven adaptive response is tumor cell survival despite cessation of EGFR signaling. Of great clinical translational importance, inhibition of the TNF adaptive response renders previously EGFR TKIresistant EGFRwt tumor cells sensitive to EGFR inhibition, suggesting that such resistant cells are still potentially oncogene addicted but are protected from EGFR TKI-induced cell death by this adaptive response. Additionally, TNF inhibition enhances the effectiveness of EGFR inhibition in EGFR-mutant NSCLC. Finally, TNF inhibition renders NSCLCs with secondary EGFR TKI resistance sensitive to EGFR inhibition. Our data suggest a key role for TNF signaling in inducing resistance to EGFR inhibition in lung cancer.

\section{Results}

EGFR inhibition leads to upregulation of TNF expression in lung cancer cell lines and xenograft tumors. Previous studies have shown that exposure of lung cancer cells to EGFR TKIs results in a rapid and biologically significant activation of NF- $\kappa \mathrm{B}$ in EGFR-mutant NSCLC cells $(19,27)$. TNF is a key activator of NF- $\kappa B(28)$, and we considered the possibility that TNF may mediate the NF- $\kappa B$ activation triggered by EGFR inhibition. Indeed, we find that exposure of lung cancer cell lines to erlotinib resulted in increased TNF mRNA levels in all 18 NSCLC cell lines examined (Supplemental Table 1; supplemental material available online with this article; https://doi.org/10.1172/JCI96148DS1) as determined by real-time quantitative PCR (qPCR) (Figure 1, A-F, and Supplemental Figure 1). Remarkably, while the temporal profiles vary, the increase in TNF is detected in both EGFRwt and EGFRmutant cell lines. The increase in TNF levels upon EGFR inhibition was confirmed at a protein level by ELISA (Figure 1, G and $\mathrm{H}$, and Supplemental Figure 2). Similar results were seen with afatinib, an irreversible EGFR inhibitor, in various cell lines (Supplemental Figure 3), including H1975 and HCC820, which harbor the EGFR T790M mutation, rendering them resistant to firstgeneration TKIs like erlotinib (Supplemental Figure 2, G and H, and Supplemental Figure 3, E and F).

Erlotinib also induced upregulation of TNF in NSCLC tumors growing in mice. Athymic mice were inoculated with EGFRmutant HCC827 and EGFRwt NSCLC A549 cells and in an EGFRwt patient-derived xenograft (PDX) model (HCC4087). Following formation of subcutaneous tumors, mice were treated with erlotinib at various time points. As is shown in Figure 1, I-N, TNF was increased in tumors upon treatment of mice with erlotinib.

EGFR activation leads to a decrease in TNF mRNA levels. The increase in TNF mRNA following EGFR inhibition suggests that either the EGFR is actively suppressing TNF levels, or the rise in TNF could be secondary to a feedback mechanism. To examine direct effects of EGFR activation, cells were treated with EGF.
This resulted in a rapid decrease in TNF mRNA and protein levels in both EGFR-mutant and EGFRwt cell lines (Figure 2, A-D, and Supplemental Figure 4, A-E). The rapid decrease in TNF mRNA suggests an effect on TNF mRNA stability rather than transcription. Also, this experiment suggests that EGFR signaling normally keeps TNF levels low and a loss of EGFR signaling results in increased TNF levels. Next, we examined whether EGFR activity influences TNF mRNA stability using actinomycin D as an inhibitor of transcription. As can be seen in Figure 2, E and F, and Supplemental Figure 4, F and G, inhibition of the EGFR with erlotinib led to an increase in TNF mRNA stability.

EGFR regulates TNF $m R N A$ via expression of $m i R-21$. $m i R-21$, an EGFR-regulated microRNA, is known to negatively regulate TNF mRNA (26, 29-31). Thus, microRNA-mediated regulation of TNF mRNA seemed like a plausible mechanism of rapid regulation of TNF mRNA stability by EGFR signaling. We first confirmed the upregulation of miR-21 by EGFR activity and its downregulation by EGFR inhibition in multiple lung cancer cell lines as shown in Figure 2, G-J, and Supplemental Figure 4, H-K. The kinetics of miR-21 regulation by EGFR inhibition is shown in Figure 2, I and J, and Supplemental Figure 4, J and K, and generally correlates with the temporal profile of TNF upregulation following EGFR inhibition. Additionally, RNA stability studies using actinomycin D demonstrated that the effect of erlotinib in enhancing TNF mRNA stability was blocked by a miR-21 mimic (Supplemental Figure 4, $\mathrm{L}$ and $\mathrm{M})$. Next, we found that inhibition of miR-21 resulted in a rescue of EGF-induced downregulation of TNF in multiple EGFR-mutant and EGFRwt cell lines (Figure 2, K and L, and Supplemental Figure 5, A-D). We confirmed miR-21 inhibition by realtime qPCR (Figure 2, M and N, and Supplemental Figure 5, E-H).

A canonical mechanism of action of microRNAs is to regulate gene expression through sequence-specific binding to the 3'-UTR of a target mRNA (32). We used a TNF 3'-UTR reporter $(33,34)$ and found that the activity of this reporter was increased by EGFR inhibition (Supplemental Figure 6, A-H). Furthermore, a miR-21 mimic compensated for the loss of miR-21 induced by erlotinib and consequently mitigated the elevated activity of the TNF 3'-UTR reporter (Supplemental Figure 6, A, C, E, G, and I) with a corresponding increase in TNF mRNA levels (Supplemental Figure 6, J-M). Importantly, an antisense miR-21 neutralized EGF-generated miR-21 to restore activity of the TNF 3'-UTR reporter (Supplemental Figure 6, B, D, F, and H) and TNF mRNA levels (Figure 2, K-N, and Supplemental Figure 5, A-H). These experiments suggest that miR-21 binds to the TNF 3 '-UTR and impairs posttranscriptional regulation of TNF mRNA.

Erlotinib-induced NF- $\kappa B$ activation is mediated by TNF. A previous study reported that NF- $\kappa \mathrm{B}$ is rapidly activated in lung cancer cells expressing EGFR activating mutations (19). Since TNF is a major activator of NF- $\mathrm{B}$, we considered the possibility that erlotinib activated NF- $\kappa B$ via an increase in TNF level. We confirmed that NF- $\kappa \mathrm{B}$ was activated by erlotinib in EGFR-mutant cell lines and found that NF-кB was also activated in EGFRwt cell lines by using a reporter assay and detecting degradation of $\mathrm{I} \kappa \mathrm{B} \alpha$ (Figure 3 , A and B). TNF receptor 1 (TNFR1) is expressed widely, while TNFR2 expression is limited to immune cells and endothelial cells $(35,36)$. We examined the effect of siRNA knockdown of TNFR1 in lung cancer cell lines. siRNA knockdown of TNFR1 led to inhibition 
A

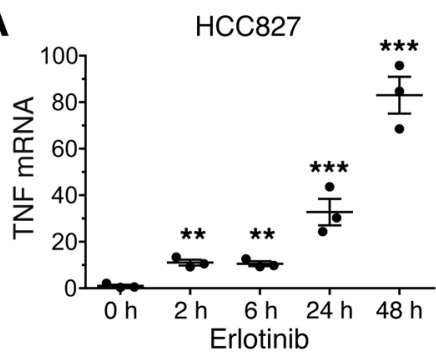

D

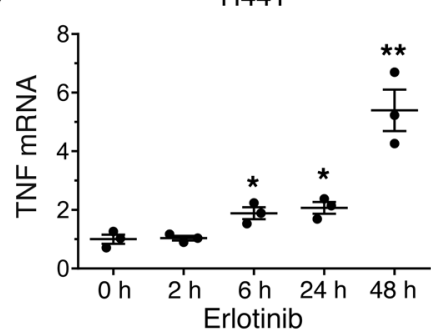

G

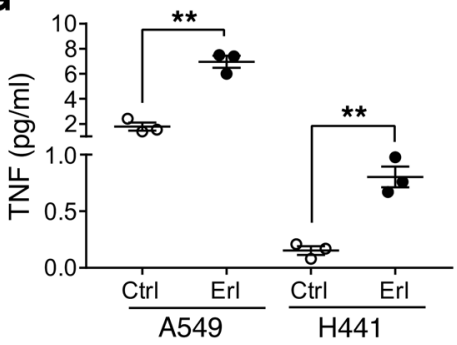

J

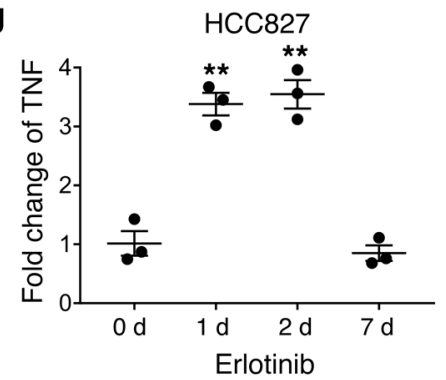

M

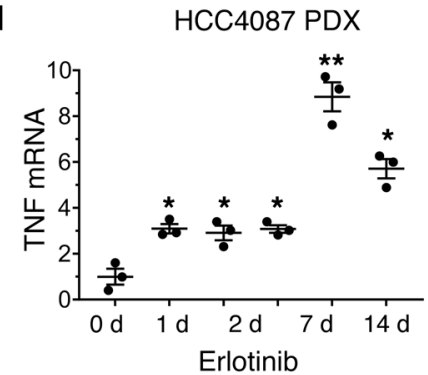

B

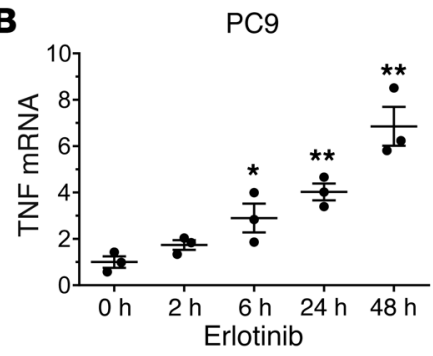

E

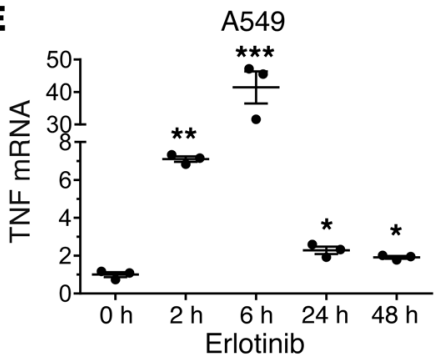

H

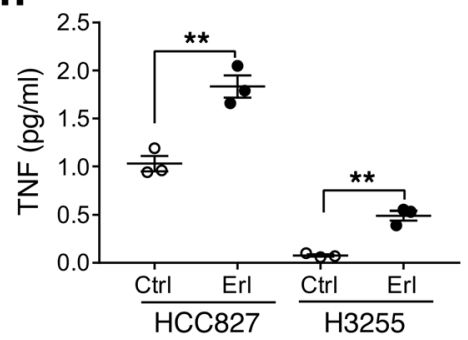

K

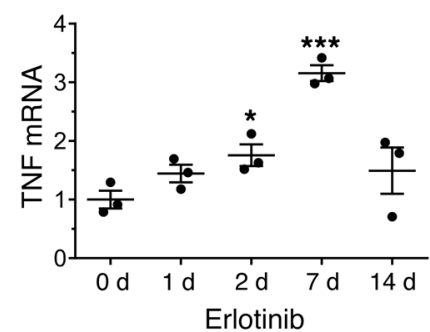

$\mathbf{N}$

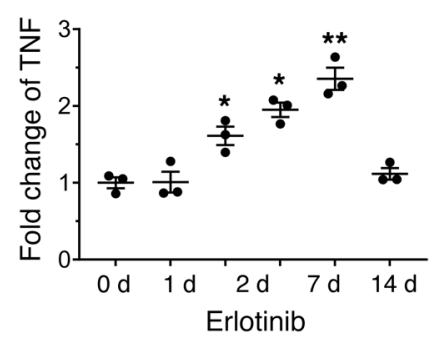

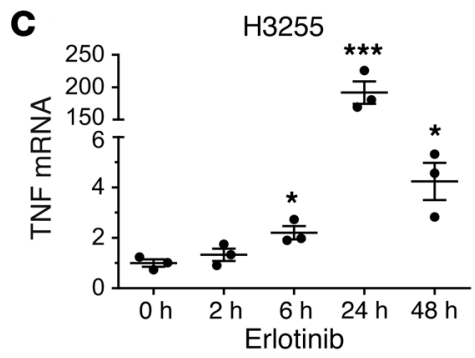

$\mathbf{F}$

Calu-3

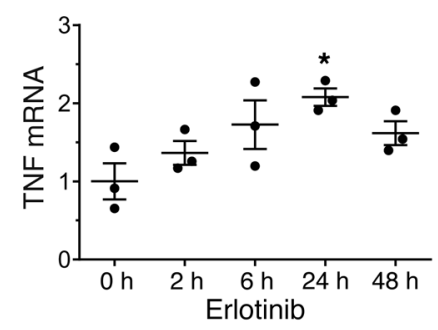

I

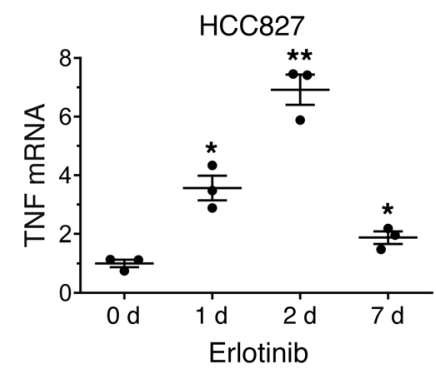

$\mathbf{L}$

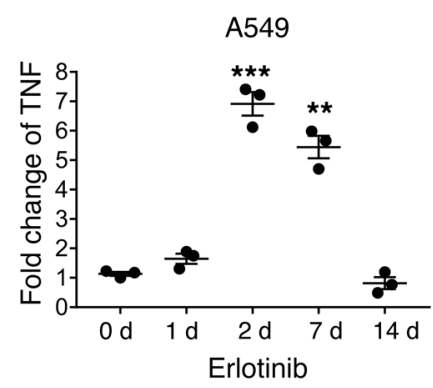

Figure 1. Upregulation of TNF signaling by EGFR inhibition. (A-F) NSCLC cell lines were cultured in RPMI-1640 in $5 \%$ FBS and were treated with erlotinib for the times indicated followed by RNA extraction and quantitative PCR (qPCR) for TNF. (G and $\mathbf{H}$ ) Cells were treated with erlotinib, and the TNF level was measured in the supernatant by ELISA. (I and J) Athymic mice were injected s.c. with HCC827 cells. After formation of tumors, erlotinib at the dose of $50 \mathrm{mg} / \mathrm{kg}$ body weight was administered for the times indicated followed by removal of tumor and quantitation of TNF mRNA by qPCR or protein by ELISA. (K and L) Athymic mice were injected s.c. with A549 cells. After formation of tumors, erlotinib at $100 \mathrm{mg} / \mathrm{kg}$ body weight was administered for the times indicated followed by removal of tumor and quantitation of TNF mRNA by qPCR or protein by ELISA. Since the TNF level remained high at 7 days in these cells, we added an additional time point at 14 days ( $n=3$ mice per group). (M and $\mathbf{N}$ ) NOD/SCID mice were implanted s.c. with HCC4087 PDX tumor tissues. After formation of tumors, erlotinib at $100 \mathrm{mg} / \mathrm{kg}$ body weight was given to the mice for $0,1,2,4,7$, and 14 days; then mice were sacrificed and tumors were removed for quantitation of TNF mRNA by QPCR or protein by ELISA ( $n=3$ mice per group). Data represent the mean \pm SEM. $n=3$ biologically independent experimental replicates $(\mathbf{A}-\mathbf{H})$ or 3 mice per group $(\mathbf{I}-\mathbf{N}) .{ }^{*} P<0.05,{ }^{* *} P<0.01,{ }^{* * *} P<0.001$, by Student's $t$ test. 
A

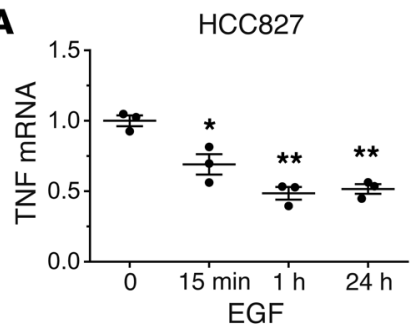

D

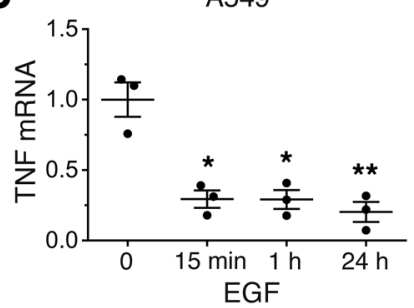

G

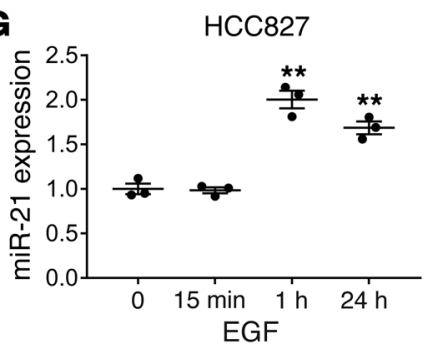

J

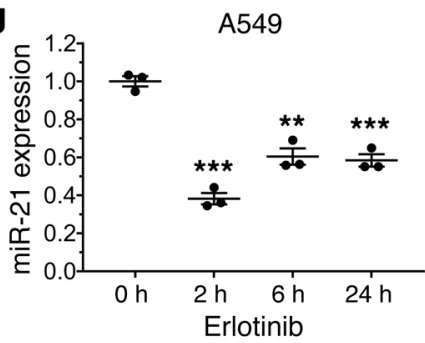

M

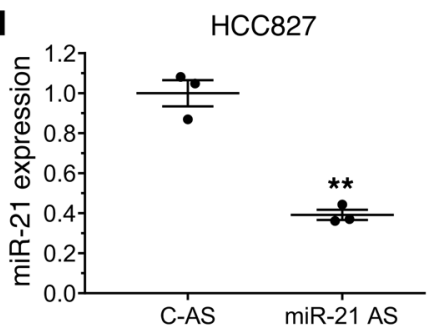

B

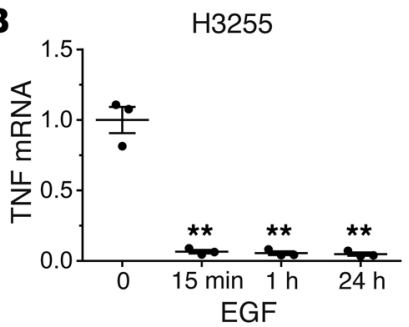

E

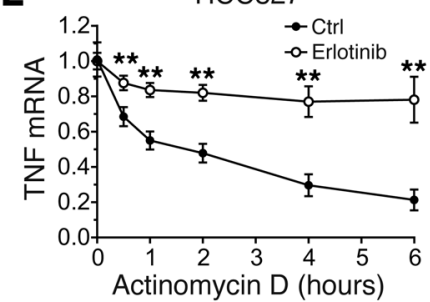

H

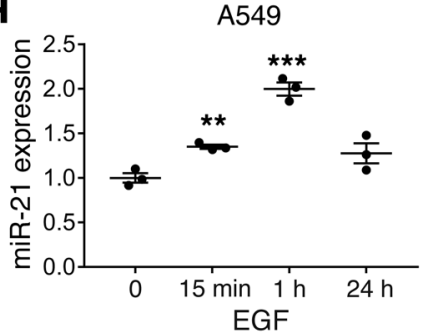

$\mathbf{K}$

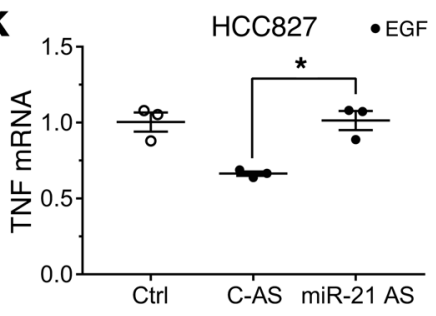

$\mathbf{N}$

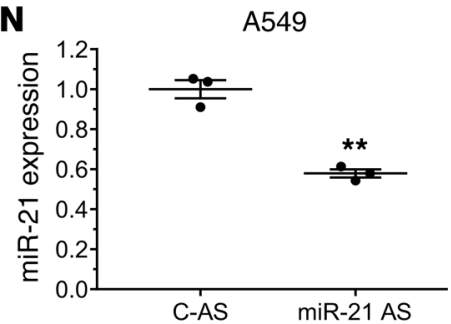

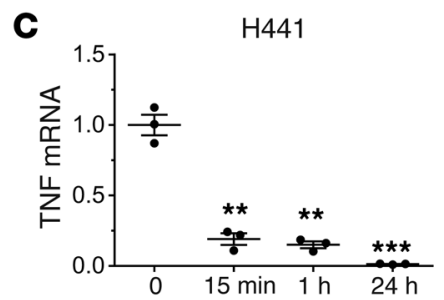

EGF

$\mathbf{F}$
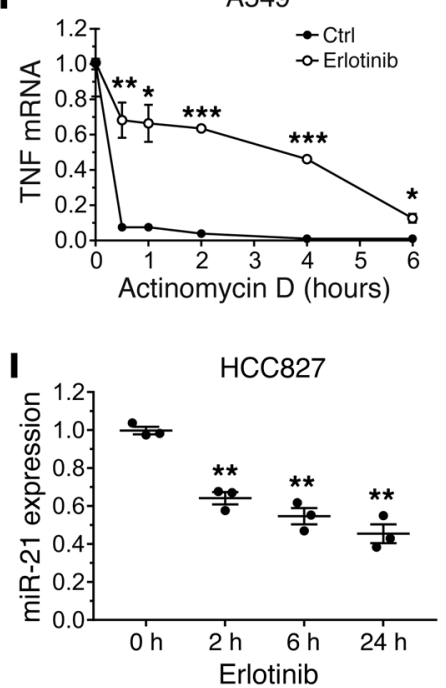

$\mathbf{L}$

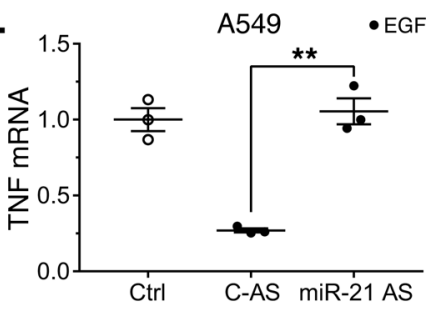

Figure 2. EGFR activity regulates TNF mRNA stability mediated by upregulation of miR-21. (A-D) NSCLC cell lines were exposed to EGF (50 ng/ml) at the indicated time points followed by qPCR for TNF mRNA. (E) HCC827 cells were treated with actinomycin D (5 $\mu \mathrm{g} / \mathrm{ml})$ and erlotinib (100 nM) for the indicated time points followed by RNA extraction and qPCR for TNF mRNA. (F) A similar experiment was done in A549 cells using an erlotinib concentration of 1 $\mu \mathrm{M}$. (G and $\mathbf{H}$ ) MiR-21 expression was examined in HCC827 and A549 cells following exposure to EGF for the indicated time points followed by qPCR using a TaqMan Human MicroRNA Assay kit. (I and J) HCC827 or A549 cells were exposed to erlotinib ( $100 \mathrm{nM}$ or $1 \mu \mathrm{M})$ for the indicated time points followed by qPCR for miR-21 using a TaqMan Human MicroRNA Assay kit. (K and L) HCC827 or A549 cells were transfected with a control antisense oligonucleotide (C-AS) or a miR-21 antisense oligonucleotide (miR-21 AS) for 48 hours followed by exposure of cells to EGF for 1 hour and qPCR for TNF. (M and N) We confirmed the downregulation of miR-21 by the miR-21 antisense oligonucleotide. In all experiments involving the use of EGF, cells were serum-starved overnight. Data represent the mean \pm SEM. $n=3$ biologically independent experimental replicates. ${ }^{*} P<0.05,{ }^{* *} P<0.01,{ }^{* * *} P<0.001$, by Student's $t$ test. 
of erlotinib-induced NF- $\kappa \mathrm{B}$ activation in both EGFR-mutant and EGFRwt cells (Figure 3, C and D, and Supplemental Figure 7, A and B). Etanercept is a fusion protein of TNFR and IgG1 and is in clinical use as a stable and effective TNF-blocking agent for rheumatologic diseases (37). Etanercept also blocked erlotinib-induced NF- $\kappa B$ activation in multiple cell lines (Figure 3, E and F, and Supplemental Figure 7, C and D). Thalidomide, a drug known to reduce TNF levels $(37,38)$, also inhibited erlotinib-induced NF- $\kappa$ B activation in both EGFRwt and EGFR-mutant cell lines (Figure 3, G and H, and Supplemental Figure 7, E and F). We confirmed that thalidomide inhibits erlotinib-induced TNF increase in lung cancer cells (Supplemental Figure 8). Thalidomide also inhibits NF-кB activation independent of its effect on TNF (39), and we confirmed that thalidomide can block NF- $\kappa \mathrm{B}$ activation induced by exogenous TNF (Figure 3, I and J). Thus, our studies indicate that erlotinib induces activation of NF- $\kappa \mathrm{B}$ via increased TNF signaling.

We recently found that EGFR inhibition results in activation of other signals such as JNK, Axl, and ERK in glioblastoma (40). However, consistent with previous studies in lung cancer cells (19), although these signals are attenuated following EGFR inhibition, neither JNK, Axl, nor ERK reactivation is detected (Supplemental Figure 9, A-D). Similarly, activation of Src and activation of FAK have been implicated in mediating resistance to EGFR inhibition in EGFR-mutant NSCLC (41). We found that EGFR inhibition led to a downregulation of both Src and FAK activation but no reactivation of these signals in multiple cell lines (Supplemental Figure 9, E and F). We did find an increase in FAK activation upon EGFR inhibition in PC9 cells (Supplemental Figure 8G) as was reported previously (41). Thus, while it is possible that Src activation and FAK activation contribute to EGFR resistance in subsets of EGFR-mutant NSCLC, they are unlikely to represent a broad mechanism of resistance to EGFR inhibition in both EGFRwt and EGFR-mutant NSCLC. In addition, we also examined the activation of YAP, since it has been implicated in mediating resistance to EGFR inhibition $(42,43)$. We found that YAP activation was increased in response to EGFR inhibition (Supplemental Figure 9, E and F). However, TNF inhibition using either etanercept or thalidomide did not block EGFR inhibition-induced YAP activation (Supplemental Figure 9, H and I). Thus, while YAP activation may be a mechanism of resistance to EGFR inhibition, TNF is not required for YAP activation and represents a distinct and unique mechanism of resistance to EGFR inhibition.

Erlotinib-induced TNF expression is regulated by $N F-\kappa B$ in a feed-forward loop. Next, we considered the possibility that erlotinibinduced increase in TNF expression is mediated by $\mathrm{NF}-\kappa \mathrm{B}$ in a feed-forward loop. We examined whether inhibition of $\mathrm{NF}-\kappa \mathrm{B}$ using a chemical inhibitor or a dominant-negative I $\mathrm{B} \alpha$ (superrepressor) mutant would block the increase in TNF following exposure of cells to erlotinib. Indeed we find that inhibition of NF- $\kappa B$ blocked the erlotinib-induced increase in TNF mRNA as detected by qPCR in both EGFRwt and EGFR-mutant cell lines (Figure 4, A, B, D, and E, and Supplemental Figure 11, D-G), while inhibition of Sp1 had no effect (Figure 4C and Supplemental Figure 11, A-C). Furthermore, blocking TNFR1 using siRNA or etanercept resulted in inhibition of erlotinib-induced TNF upregulation (Figure 4, F-J). These data indicate that TNF is upregulated via a feed-forward loop that requires activity of NF- $\mathrm{KB}$ and TNF signaling.
Finally, we found that NF- $\mathrm{BB}$ could bind to 2 putative sites on the TNF promoter (Supplemental Figure 10) by chromatin immunoprecipitation coupled with qPCR (ChIP-qPCR) assay. While the binding of NF- $\kappa \mathrm{B}$ p 65 subunit to the TNF promoter was quite low under basal conditions, when EGFR was inhibited there was increased presence of NF- $\kappa \mathrm{B}$ on the TNF promoter in both EGFRwt and EGFR-mutant cells (Figure 4K and Supplemental Figure 12, A-C).

TNF inhibition sensitizes lung cancer cells to EGFR inhibition. TNF is a key mediator of the inflammatory response and inflammation-induced cancer $(28,44,45)$. Depending on the cellular context, it may play a role in cell death or in cell survival. We hypothesized that increased TNF secretion protects EGFR-expressing lung cancer cells from cell death following the loss of EGFR signaling. We started with EGFRwt-expressing A549 and H441 cells, which are known to be resistant to EGFR TKIs. First, we did siRNA knockdown of TNFR1 and found that this conferred sensitivity to erlotinib in cell survival assays. Erlotinib alone or TNFR1 silencing alone had no effect (Figure 5, A-C). Next, we examined the effect of thalidomide, an inhibitor of TNF and of NF- $\kappa$ B activation. Thalidomide alone had no effect, but it rendered A549 and H441 cells sensitive to the effects of erlotinib (Figure 5, D and E). Thus EGFR inhibition combined with either biological or chemical inhibition of TNF signaling renders EGFRwt-expressing resistant cells sensitive to EGFR inhibition. Etanercept also rendered both A549 and H441 cells sensitive to the effect of erlotinib (Figure 5, F and G), whereas etanercept alone had no effect. We also found that combining etanercept or thalidomide with afatinib $(1 \mu \mathrm{M})$ impacted cell viability (Figure 5, H-K). A colony formation assay confirmed that TNF inhibition rendered EGFRwt-expressing resistant lines sensitive to erlotinib (Figure 5, L and M, and Supplemental Figure 13A). TNF inhibition also rendered EGFRwt-expressing cell lines sensitive to afatinib (Supplemental Figure 13, B-E).

Next we examined the effect of combining TNF and EGFR inhibition in sensitive lung cancer cells with EGFR activating mutations (HCC827, EGFR exon 19 deletion; or H3255, EGFR L858R mutation). Experiments with low concentrations of erlotinib revealed a sensitizing effect of TNF inhibition obtained with TNFR1 gene silencing (Figure 6, A-C) or with etanercept or thalidomide (Figure 6, D-G), while TNF inhibition alone had no effect. We also tested a combination of afatinib and thalidomide or etanercept and found a greater sensitivity to EGFR inhibition (Figure 6, H-K). A colony formation assay confirmed that TNF inhibition enhanced sensitivity of EGFR-mutant lines to erlotinib (Figure 6, L and M, and Supplemental Figure 13A). Additional NSCLC lines with EGFRwt (Calu-3 and H1373) exhibited similar results (Supplemental Figure 14, A and B).

Since we hypothesize that erlotinib-induced TNF expression mediates resistance to EGFR inhibition, we examined whether exogenous TNF would protect cells from erlotinib-induced cell death observed in EGFR oncogene-addicted lines. Indeed, we find that exogenous TNF protected HCC827 and H3255 cells from erlotinib-induced cell death, as shown in Figure 6, $\mathrm{N}$ and $\mathrm{O}$.

Inhibition of $N F-\kappa B$ enhances sensitivity to EGFR inhibition. Previous studies have shown that NF- $\kappa B$ plays a role in resistance to EGFR inhibition in EGFR-mutant cells $(19,27)$. We found that chemical inhibition of NF- $\kappa \mathrm{B}$ rendered EGFRwt-expressing cell lines sensitive to EGFR inhibition (Figure 7, A-D, and Supplemen- 


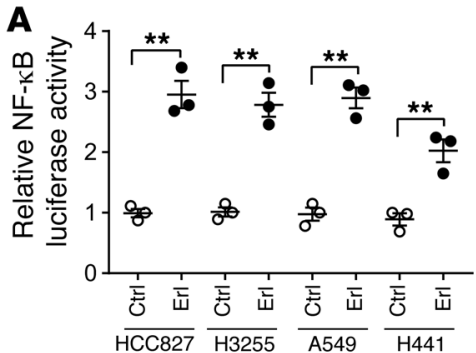

B
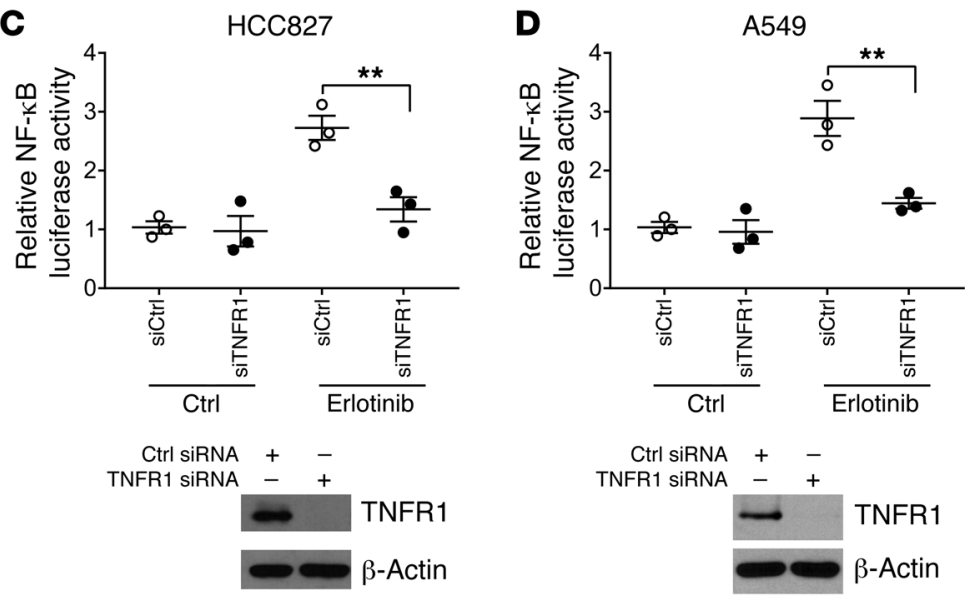

E

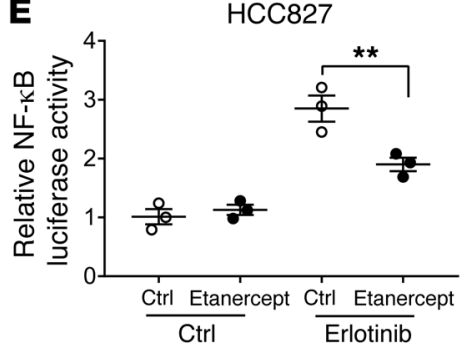

$\mathbf{F}$
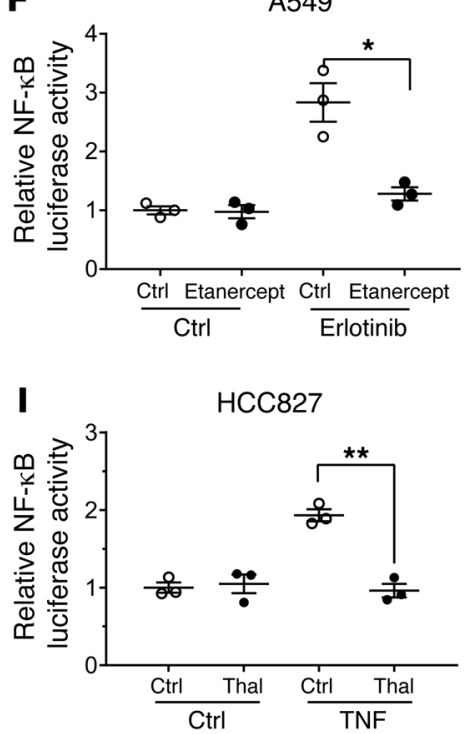

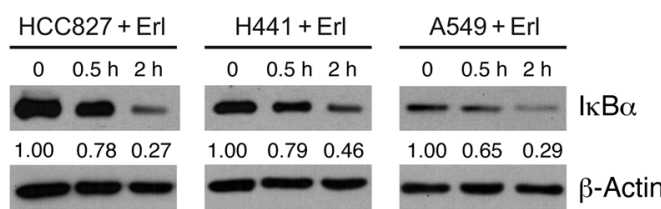

Ctrl SiRNA +TNFR1 SIRNA - +

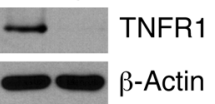

G

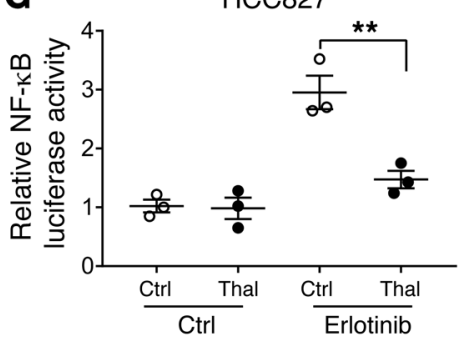

J

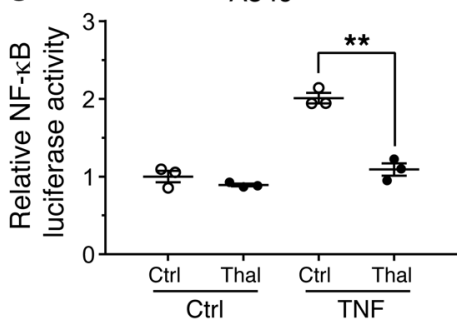

H

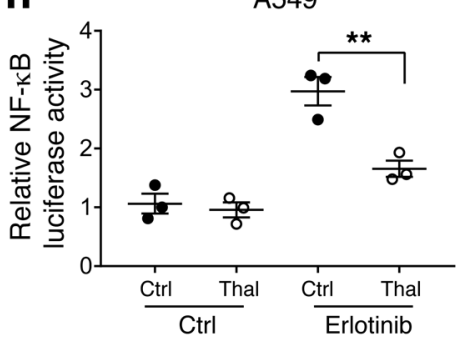

Figure 3. EGFR inhibition induces a TNF-dependent activation of NF-кB. (A) HCC827, H3255, A549, and H441 cells were exposed to erlotinib (100 nM for EGFR-mutant and $1 \mu \mathrm{M}$ for EGFRwt cells) for 24 hours followed by a dual luciferase reporter assay. Renilla luciferase was used as an internal control. (B) Cells were treated with erlotinib at various time points followed by preparation of cell lysates and Western blot with an IкB $\alpha$ antibody. Western blots are representative of at least 3 independent replicates. The quantified values reflect the ratios of IKB $\alpha / a c t i n$. (C) siRNA knockdown of TNFR1 was performed in HCC827 cells followed by transfection of cells with an NF- $\kappa B$ luciferase reporter and exposure of cells to erlotinib, followed by a reporter assay. Silencing of TNFR1 was confirmed with a Western blot. (D) A similar experiment was undertaken in A549 cells, and TNFR1 silencing was confirmed with a Western blot. Western blots shown in $\mathbf{C}$ and $\mathbf{D}$ are representative of at least 3 independent replicates. (E) The TNF-blocking drug etanercept was used at a concentration of $100 \mu \mathrm{g} / \mathrm{ml}$ along with erlotinib for 24 hours followed by a reporter assay in HCC827 cells. (F) A similar experiment was conducted in A549 cells. (G and $\mathbf{H}$ ) Reporter assay for NF- $\mathrm{KB}$ in cells treated with erlotinib in the presence or absence of thalidomide ( $5 \mu \mathrm{g} / \mathrm{ml})$ for 24 hours. (I and J) HCC827 and A549 cells were treated with exogenous TNF $(10 \mathrm{ng} / \mathrm{ml})$ with or without thalidomide for 24 hours followed by a reporter assay for NF- $\mathrm{KB}$ transcriptional activity. In luciferase assays, cells were transfected with reporter 24 hours before exposure to erlotinib. Data represent the mean \pm SEM. $n=3$ biologically independent experimental replicates ( $\mathbf{A}$ and $\mathbf{C}-\mathrm{J}) .{ }^{*} P<0.05,{ }^{* *} P<0.01$, by Student's $t$ test. Erl, erlotinib; Thal, thalidomide. 
A

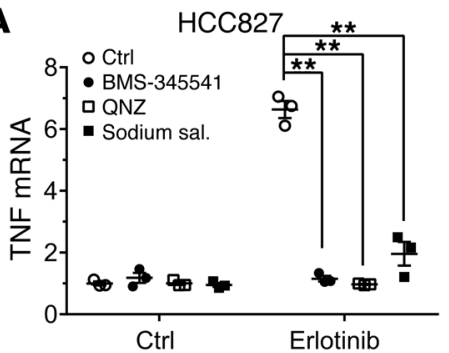

D

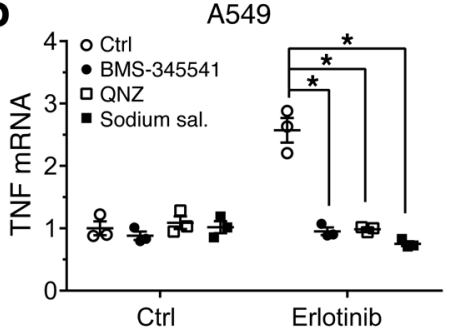

$\mathbf{F}$
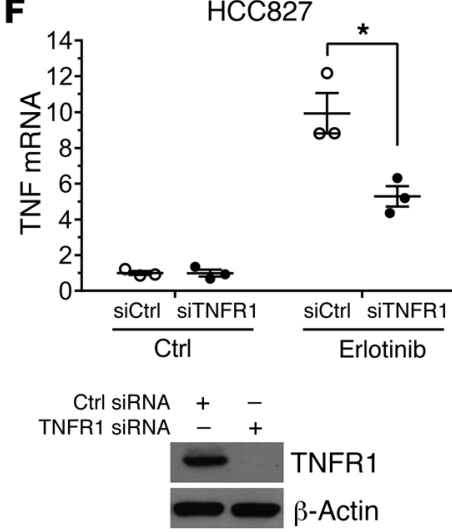

I

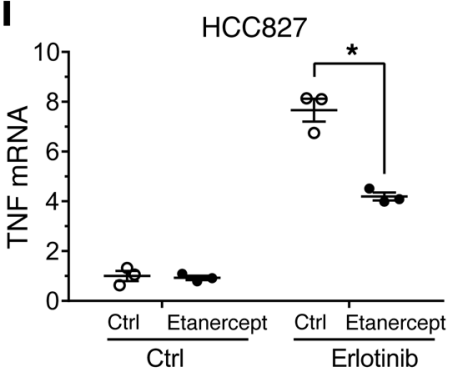

B

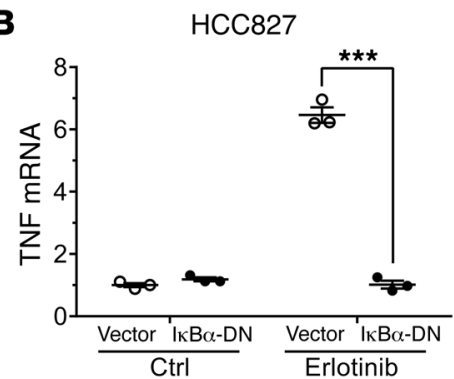

E

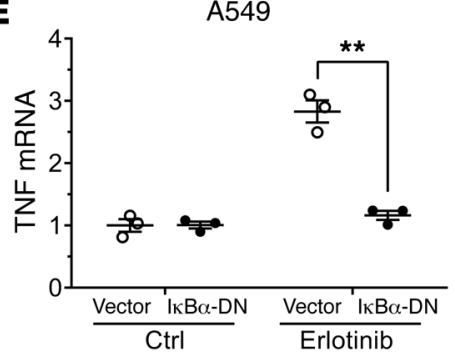

G
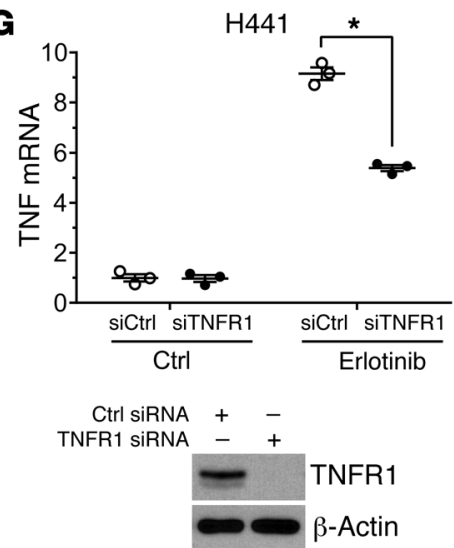

J

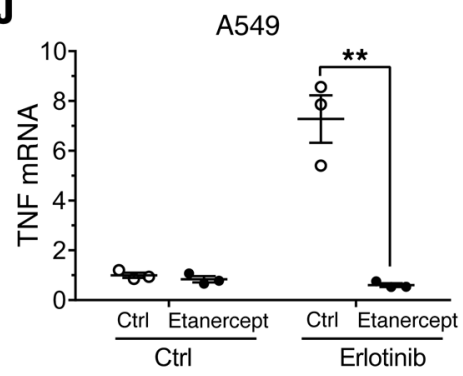

C

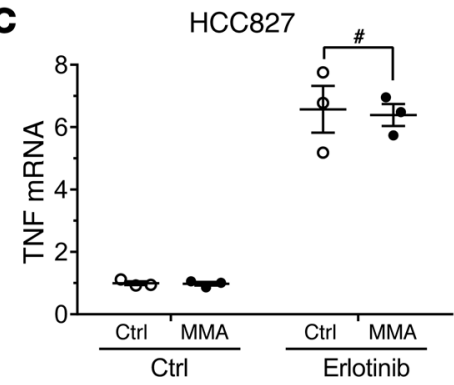

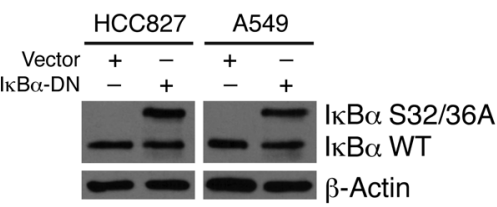

H
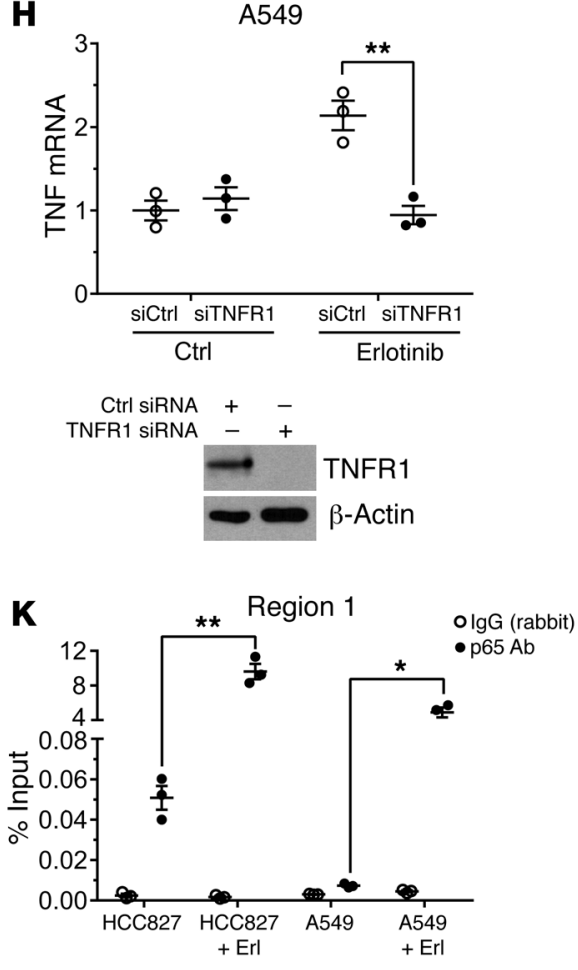

Figure 4. A TNF/NF-KB feed-forward loop regulates the expression of TNF in response to EGFR inhibition. (A) Inhibition of NF- $\kappa B$ using various chemical inhibitors - BMS-345541 (100 nM), 6-amino-4-(4-phenoxyphenylethylamino) quinazoline (QNZ; $1 \mu \mathrm{M})$, or sodium salicylate (5 mM) - inhibited erlotinibinduced upregulation of TNF in HCC827 cells as determined by QPCR. Cells were treated with NF- $\mathrm{kB}$ inhibitors for 1 hour and then $100 \mathrm{nM}$ erlotinib for 24 hours. (B) Expression of a dominant-negative (DN) IKB $\alpha$ super-repressor mutant blocks erlotinib-induced upregulation of TNF in HCC827 cells. (C) Mithramycin A (MMA) (1 $\mu \mathrm{M})$, an inhibitor of Sp1, failed to inhibit erlotinib-induced TNF upregulation. ( $\mathbf{D}$ and $\mathbf{E})$ The same experiment as in $\mathbf{A}$ and $\mathbf{B}$ was conducted in $\mathrm{A} 549$ cells. Expression of the dominant-negative I $\kappa \mathrm{B} \alpha$ super-repressor mutant was detected by Western blot. The mutant protein migrates more slowly on electrophoretic gels. (F-H) siRNA knockdown of TNFR1 in NSCLC cells inhibits erlotinib-induced upregulation of TNF mRNA as detected by qPCR. Silencing of TNFR1 was confirmed by Western blot. (I and J) Inhibition of TNFR signaling using etanercept (100 $\mu \mathrm{g} / \mathrm{ml})$ results in a block of erlotinib-induced TNF upregulation in HCC827 and A549 cells. (K) ChIP was carried out to assess the recruitment of the NF- $\kappa B$ p65 subunit onto the TNF promoter using primers specific to NF- $\mathrm{KB}$ binding region 1 on the TNF promoter. There is a substantially increased p65 antibody enrichment (percentage of input, compared with rabbit IgC) on the TNF promoter in both HCC827 and A549 cells in response to erlotinib treatment for 24 hours. Data represent the mean \pm SEM. $n=3$ biologically independent experimental replicates. ${ }^{*} P<0.05,{ }^{* *} P<0.01$, ${ }^{* * *} P<0.001$, not statistically significant, by Student's $t$ test for comparing 2 indicated groups, or 1-way ANOVA, Dunnett's method, for comparing multiple groups with the same control (A and $\mathbf{D})$. Western blot results are representative of at least 3 independent replicates. 
A

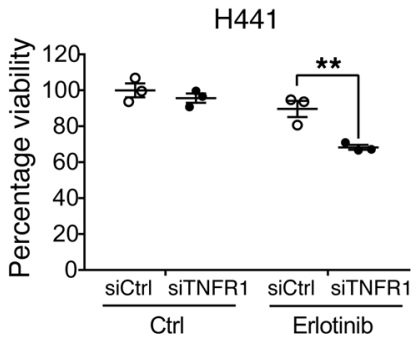

D

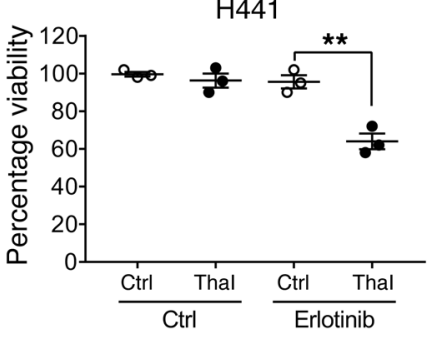

G

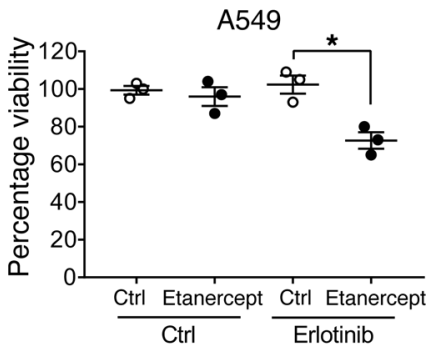

J

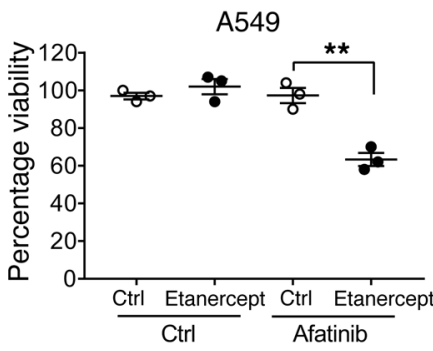

M

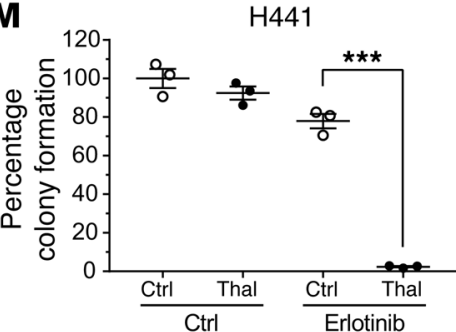

B

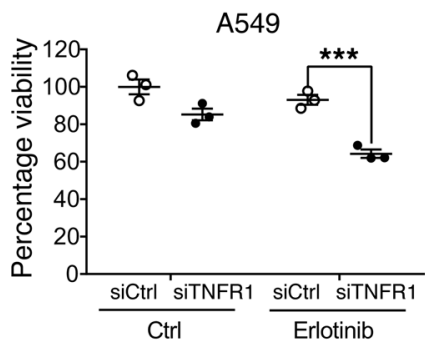

E

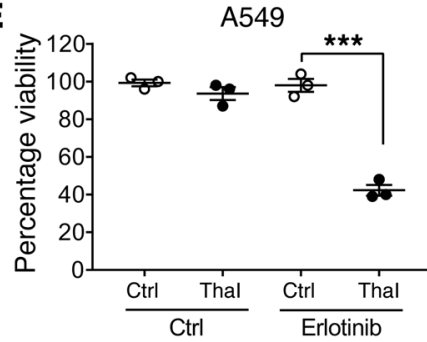

H

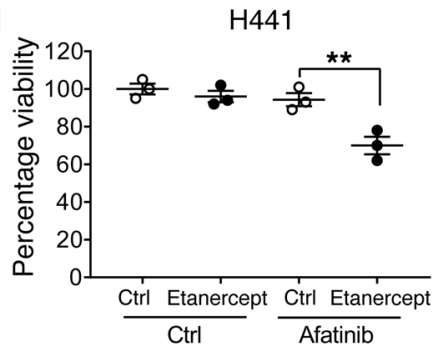

$\mathbf{K}$

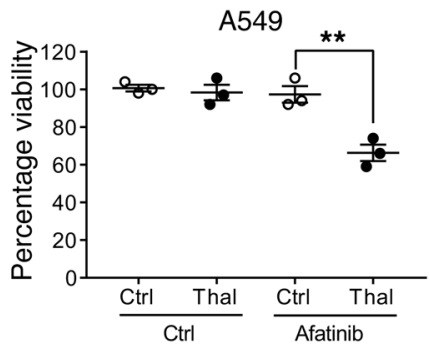

C

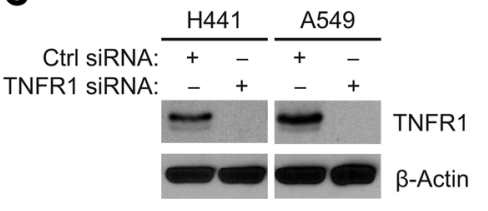

$\mathbf{F}$

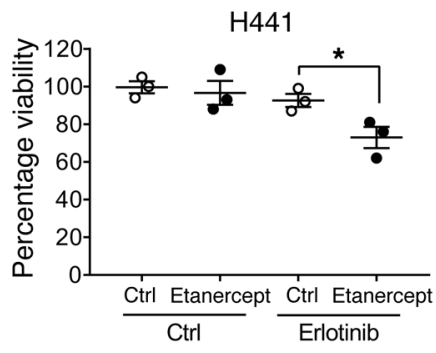

I

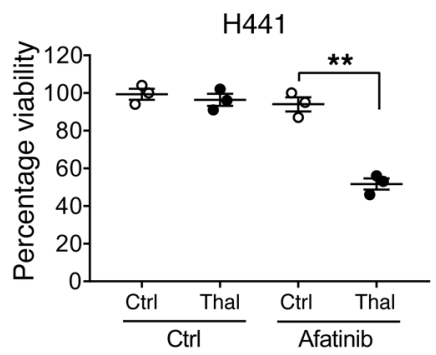

$\mathbf{L}$

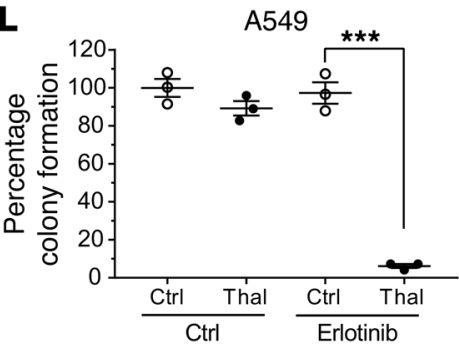

Figure 5. Inhibition of TNF induces sensitivity of EGFRwt-expressing NSCLC cells to EGFR inhibition. (A and B) AlamarBlue cell viability assay in H441 or A549 cells. TNFR1 was silenced by siRNA transfection for 48 hours, and cells were exposed to erlotinib $(1 \mu \mathrm{M})$ for 72 hours in RPMI-1640 with $5 \%$ FBS. (C) Silencing of TNFR1 was confirmed by Western blot. Western blot results are representative of at least 3 independent replicates. (D and E) Thalidomide sensitizes H441 and A549 cells to EGFR inhibition with erlotinib. Thalidomide $(5 \mu \mathrm{g} / \mathrm{ml})$ and erlotinib were added to $\mathrm{H} 441$ and $\mathrm{A} 549$ cells concurrently, and AlamarBlue assay was done after 72 hours. (F and $\mathbf{G})$ A similar experiment was done using etanercept $(100 \mu \mathrm{g} / \mathrm{ml})$ and erlotinib in $\mathrm{H} 441$ and A549 cells. (H) H441 cells were treated with afatinib $(1 \mu \mathrm{M})$ in the presence or absence of etanercept. AlamarBlue assay was conducted after 72 hours. (I) H441 cells were treated with afatinib and thalidomide for 72 hours, followed by AlamarBlue assay. (J and $\mathbf{K}$ ) Similar experiments were done as described in $\mathbf{H}$ and $\mathbf{I}$ in A549 cells. ( $\mathbf{L}$ and $\mathbf{M}$ ) EGFRwt cells were seeded in 6 -well plates at 1,000 cells per well, and incubated with $20 \mu \mathrm{g} / \mathrm{ml}$ thalidomide and/or $1 \mu \mathrm{M}$ erlotinib. Fourteen days later, cell colonies were fixed by $100 \%$ methanol and then stained by $0.5 \%$ crystal violet in $25 \%$ methanol. Images were captured by a scanner, and colony counts were processed by Image). Data represent the mean percentage of control \pm SEM. $n=3$ biologically independent experimental replicates (A, B, and D-M). ${ }^{*} P<0.05,{ }^{* *} P<0.01,{ }^{* * *} P<0.001$, by Student's $t$ test. 
tal Figure 14, E-H). We also confirmed that inhibition of NF- $\kappa B$ enhanced sensitivity of oncogene-addicted cells to EGFR inhibition (Figure 7, C and D, and Supplemental Figure 14, G and H). Finally, we find that overexpressing the $\mathrm{p} 65$ subunit of $\mathrm{NF}-\kappa \mathrm{B}$ resulted in a resistance to exposure of lung cancer cells with EGFR activating mutations to EGFR inhibition as shown in Figure 7, E-G, suggesting that NF- $\kappa \mathrm{B}$ activation impairs sensitivity to EGFR inhibition.

Lymphotoxin- $\beta$ is a key effector of $T N F / N F-\kappa B$-induced resistance to EGFR inhibition. To elucidate mechanisms that mediate the biological effects of $\mathrm{TNF} / \mathrm{NF}-\kappa \mathrm{B}$ regulation, we undertook an analysis of NF- $\kappa \mathrm{B}$ target genes induced by EGFR inhibition in lung cancer cells, using a human NF- $\kappa$ B signaling pathway RT ${ }^{2}$ Profiler PCR Array (Qiagen) that evaluates the expression of 84 key NF- $\mathrm{B}$ target genes. Twenty-nine of the $84 \mathrm{NF}-\kappa \mathrm{B}$ target genes in this PCR array were increased twofold or greater, including TNF (Figure $7 \mathrm{H}$ and Supplemental Figure 14I). Next, we investigated whether individual components of the NF- $\kappa$ B target signature genes play a key role in mediating resistance to EGFR inhibition. Lymphotoxin- $\beta$ (LTB, TNFC), a member of the TNF superfamily, has a known role in cancer development, including in solid tumors (46), and may play an important role in mediating resistance to cetuximab in head and neck cancer (47). Lymphotoxin- $\beta$ may trigger multiple survival mechanisms, including activation of NF- $\kappa \mathrm{B}(46)$, acceleration of Akt-induced cancer (48), and enhanced EGFR signaling (47). The induction of lymphotoxin- $\beta$ following EGFR inhibition is particularly robust (25.51-fold) (Figure $7 \mathrm{H}$ ). We confirmed the erlotinib-induced increase in lymphotoxin- $\beta$ using qPCR (Figure 7, I and J, and Supplemental Figure 15, A and B) and by ELISA (Supplemental Figure 15, E-H). An increase in lymphotoxin- $\beta$ in response to EGFR inhibition was also detected in mouse tumors (Supplemental Figure 17, D-F). We confirmed that the increase in lymphotoxin- $\beta$ that results from EGFR inhibition can be blocked by both TNF and NF- $\mathrm{KB}$ inhibition (Figure 7, I-L, and Supplemental Figure 15, A-H). Importantly, we find that the biological effects of concomitant EGFR inhibition and siRNA knockdown of lymphotoxin $\beta$ were similar to those of TNF or NF- $\kappa B$ inhibition and resulted in enhanced sensitivity of lung cancer cells to EGFR inhibition (Figure 7, M-P, and Supplemental Figure 15, I-L). These data indicate that lymphotoxin- $\beta$ induction is mediated by TNF and NF- $\mathrm{KB}$ signaling in the context of EGFR inhibition and that lymphotoxin- $\beta$ may be a key mediator of resistance to EGFR inhibition in lung cancer cells.

Combined inhibition of TNF and EGFR in an animal model of lung cancer. Next, we examined whether a combined inhibition of TNF and EGFR would influence sensitivity to erlotinib in mouse xenograft models. Since our studies indicated that a TNF/NF- $\mathrm{B}$ loop was a key mediator of resistance to EGFR inhibition, we chose thalidomide for our initial studies. A number of studies have demonstrated that thalidomide downregulates TNF levels and also inhibits NF- $\mathrm{BB}$ activation directly. A549 cells (EGFRwt) were injected into the flanks of mice to form subcutaneous tumors. Once tumors became visible, treatment was started with control vehicle, erlotinib, thalidomide, or erlotinib plus thalidomide as indicated in Figure 8A. As expected, we found robust tumor growth in controls. The groups treated with erlotinib or with thalidomide alone had a minor decrease in tumor growth that was not statistically significant. However, combined inhibition of erlotinib and thalid- omide resulted in a highly effective suppression of tumor growth (Figure 8A). We also found the combination of erlotinib and thalidomide to be highly effective in inhibiting the growth of an EGFRwt PDX tumor (Figure 8B). Additionally, we examined the effect of a combined TNF and EGFR inhibition in a mouse subcutaneous model using EGFR-mutant erlotinib-sensitive HCC827 cells and found that the combination of EGFR inhibition plus thalidomide resulted in a more effective inhibition of tumor growth than EGFR inhibition alone while thalidomide alone had no significant effect (Figure 8C). Next, to definitively determine the role of TNF, we examined the effect of stably silencing TNF using shRNA lentivirus. Effective silencing of TNF was determined by decreased basal levels and a lack of TNF upregulation in response to LPS by qPCR and ELISA (Figure 8D and Supplemental Figure 16A). We also confirmed that TNF-silenced clones were more sensitive to EGFR inhibition in cell viability assays (Supplemental Figure 16, B and C). Next, we determined the effect of EGFR inhibition in A549 cells with stably silenced TNF in a mouse subcutaneous model. As can be seen in Figure 8E, stable silencing of TNF resulted in enhanced sensitivity of xenografted tumors to afatinib. We also found that etanercept rendered A549 cells sensitive to the effect of EGFR inhibition (Figure 8F). Also, in addition to the increase in TNF levels (Figure 1, I-N), we confirmed activation of NF- $\mathrm{B}$ by detecting nuclear localization of the p65 subunit of NF- $\kappa B$ in erlotinib-treated tumors (Supplemental Figure 17, A and B), as well as degradation of I $\mathrm{B} \alpha$ (Supplemental Figure 17C). We noted a downregulation of phosphorylated ERK (p-ERK) with erlotinib, while p-Akt was largely unaffected in mouse tumors (Supplemental Figure 17C). We also detected erlotinib-induced upregulation of NF- $\mathrm{BB}$ target genes in tumor tissue (Supplemental Figure 17, $\mathrm{D}-\mathrm{I})$; in addition, we detected the presence of apoptotic cells in untreated or treated A549, HCC827, and PDX tumors by TUNEL assay, and cell proliferation by Ki67 staining in formalin-fixed, paraffin-embedded tissues from these tumors. An increase in apoptosis could be detected when a combination of erlotinib plus thalidomide was used (Supplemental Figure 18, A and B). A lesser degree of apoptosis could also be detected in HCC827-derived tumors treated with erlotinib alone. Proliferation was suppressed when a combination of erlotinib plus thalidomide was used and also, to a lesser degree, when erlotinib alone was used in HCC827 tumors (Supplemental Figure 18, C and D).

To further examine the effect of combined EGFR plus TNF inhibition in an immunocompetent model, we used a well-established transgenic mouse model of lung cancer that is driven by doxycycline-mediated induction of the EGFR L858R mutation (49). Once tumors were detected by imaging, treatment was started with control vehicle, erlotinib, thalidomide, or erlotinib plus thalidomide as indicated in Figure 9, A and B. As expected, we found robust tumor growth in controls. Thalidomide alone did not have a significant effect on tumor growth. However, the combination of erlotinib plus thalidomide induced a more effective inhibition of tumor growth than erlotinib alone, demonstrating that EGFR plus TNF inhibition is also effective in an immunocompetent model.

A combination of EGFR plus TNF inhibition confers sensitivity to EGFR-mutant lung cancer cells with acquired resistance to erlotinib. We examined whether HCC827 lines rendered experimentally resistant to EGFR inhibition $(11,50)$ could be rendered sensitive 
A

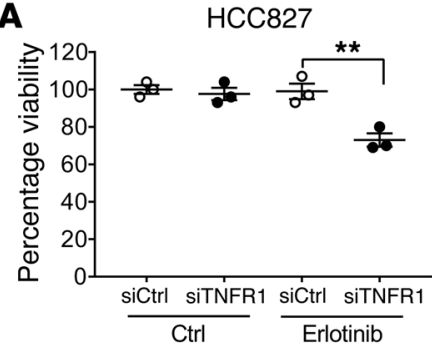

D

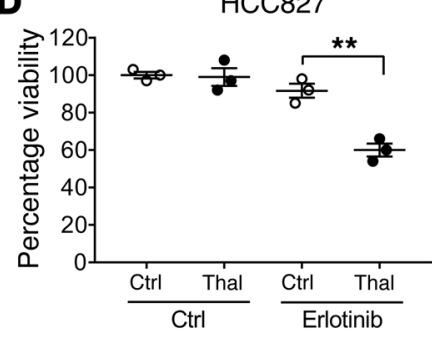

G

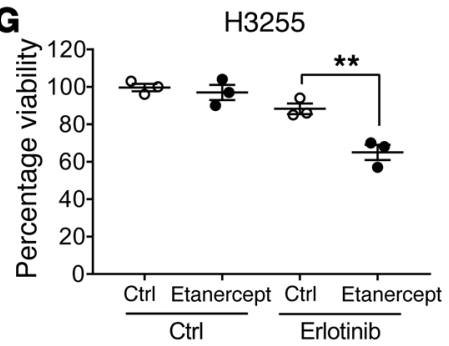

J

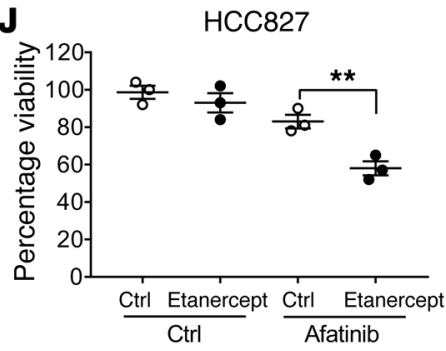

M

H3255

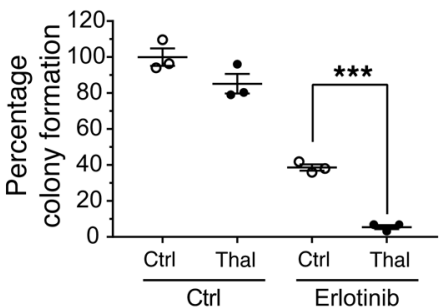

B

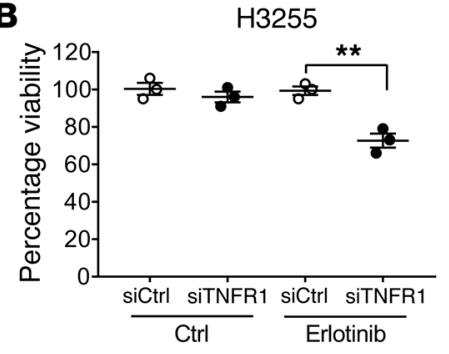

E

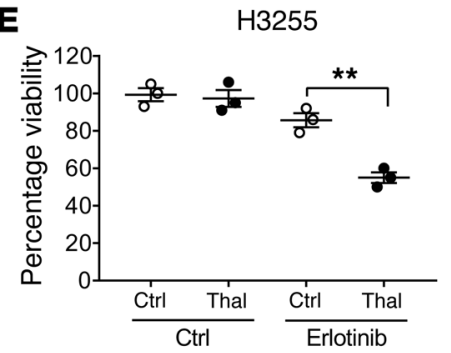

H

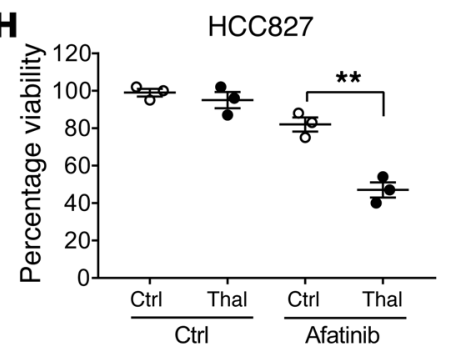

K

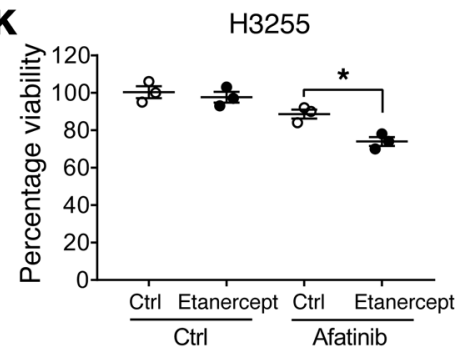

$\mathbf{N}$

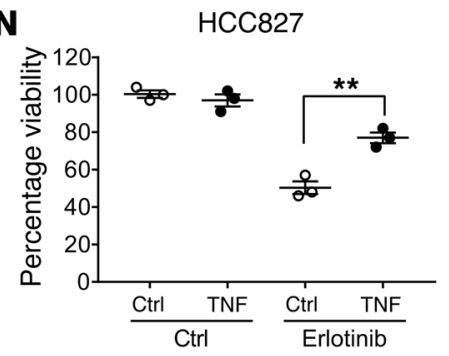

C

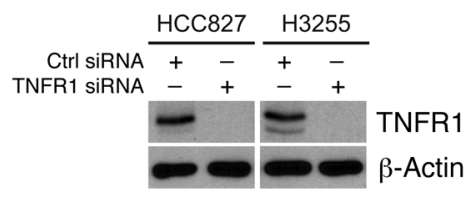

$\mathbf{F}$

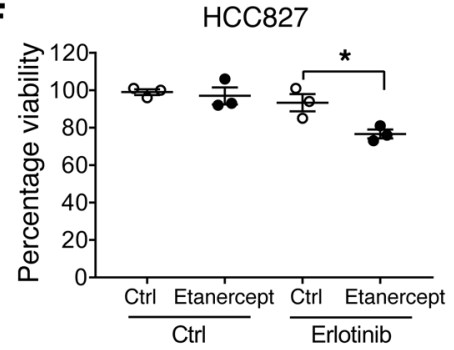

I

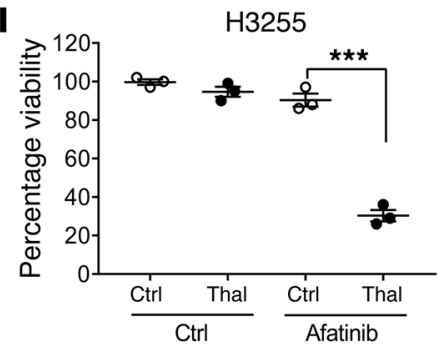

$\mathbf{L}$

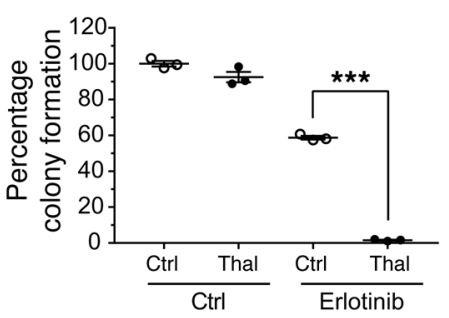

o

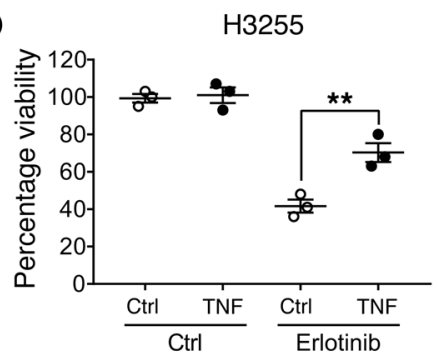

Figure 6. Inhibition of TNF enhances sensitivity of NSCLC cells with EGFR activating mutations to EGFR inhibition. (A and B) AlamarBlue assay in HCC827 or H3255 cells. TNFR1 was silenced using siRNA, and 48 hours later cells were exposed to erlotinib for 72 hours. (C) Silencing of TNFR1 was confirmed by Western blot. Results are representative of 3 independent replicates. ( $\mathbf{D}$ and $\mathbf{E})$ Thalidomide sensitizes HCC 827 and H3255 cells to EGFR inhibition. Thalidomide ( $5 \mu$ g/ $\mathrm{ml}$ ) and erlotinib were added concurrently, and AlamarBlue assay was done after 72 hours. (F and $\mathbf{G})$ Similar experiments were done using etanercept (100 $\mu \mathrm{g} /$ $\mathrm{ml}$ ) and erlotinib in HCC827 and H3255 cells. (H and I) HCC827 and H3255 cells were treated with afatinib with or without thalidomide for 72 hours, followed by AlamarBlue assay. ( $\mathbf{~ a n d} \mathbf{K}$ ) Similar experiments were performed in HCC 827 and $\mathrm{H} 3255$ cells with afatinib and etanercept. The concentration of erlotinib or afatinib was $10 \mathrm{nM}$ in A-K. (L and M) EGFR-mutant NSCLC cells were seeded in 6 -well plates at 1,000 cells per well, and incubated with $5 \mu \mathrm{g} / \mathrm{ml}$ thalidomide and/or erlotinib $1 \mathrm{nM}$. Fourteen days later, cell colonies were fixed by $100 \%$ methanol and then stained by $0.5 \%$ crystal violet in $25 \%$ methanol. Images were captured by a scanner, and colony counting was processed by Imagej. Data are presented as the average percentage of untreated colonies \pm SEM from 3 experiments. (N and $\mathbf{0}$ ) Exogenous TNF protects H3255 and HCC827 from all erlotinib-induced cell death. Cells were exposed to erlotinib (100 nM) with or without TNF $(1 \mathrm{ng} / \mathrm{ml})$. Cell viability was determined 72 hours later using AlamarBlue assay. Data represent the mean percentage of control \pm SEM. $n=3$ biologically independent experimental replicates $(\mathbf{A}, \mathbf{B}$, and $\mathbf{D}-\mathbf{0}) .{ }^{*} P<0.05,{ }^{* *} P<0.01,{ }^{* * *} P<0.001$, by Student's $t$ test. 
A

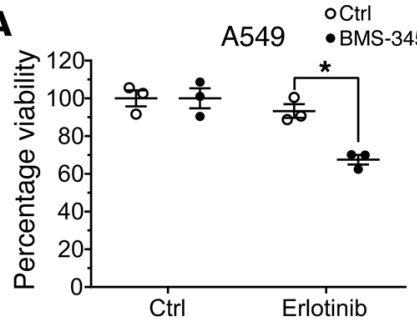

D

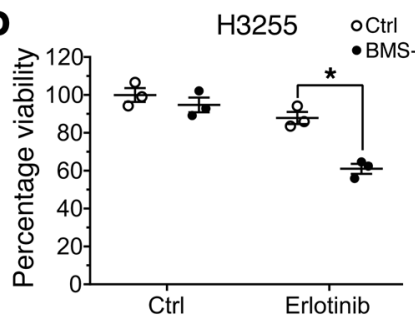

H

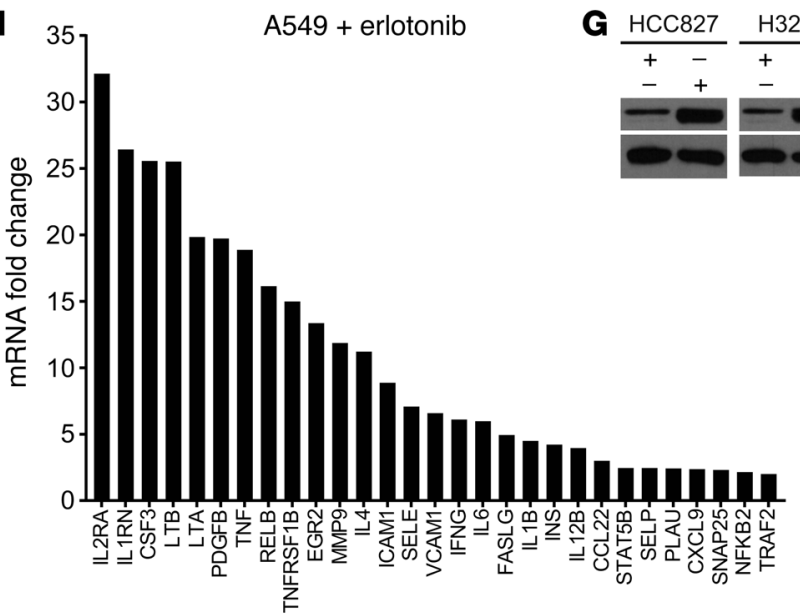

$\mathbf{K}$

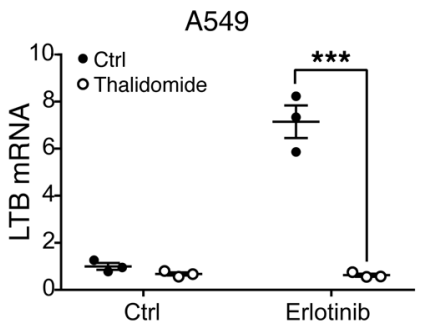

$\mathbf{N}$

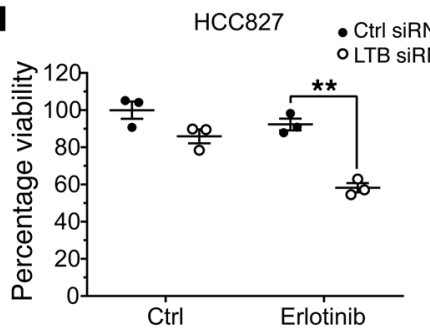

$\mathbf{L}$
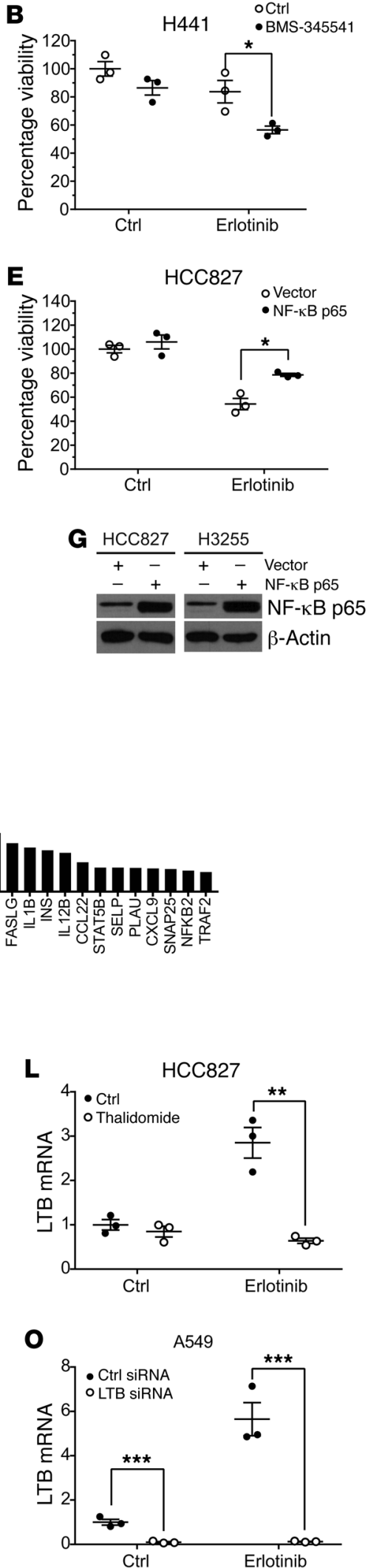

I
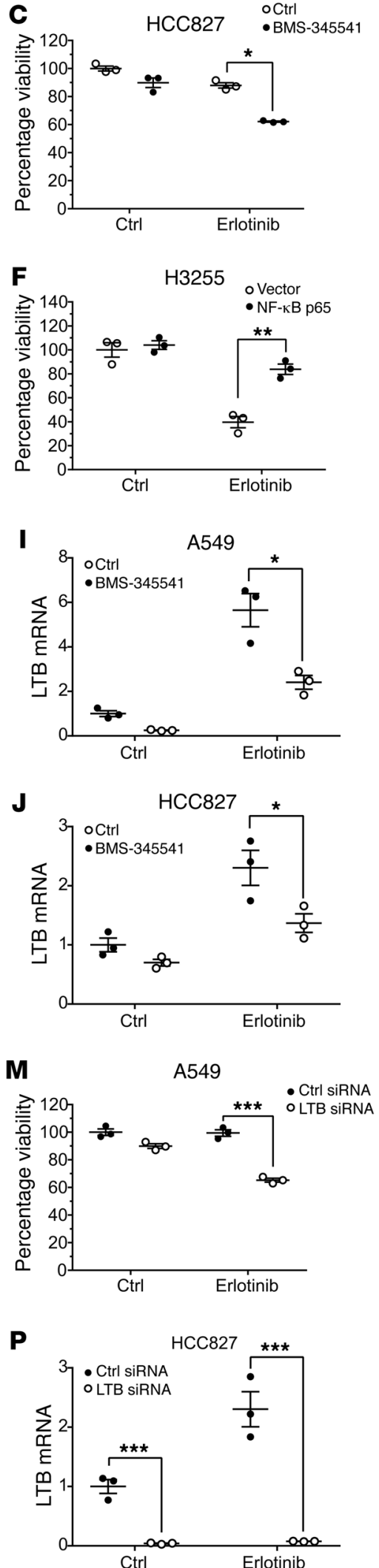
Figure 7. Inhibition of NF- $\kappa B$ sensitizes EGFRwt and EGFR-mutant NSCLC to EGFR inhibition. (A-D) NSCLC cells were exposed to erlotinib with or without NF-kB inhibitor BMS-345541 (100 nM) for 72 hours followed by an AlamarBlue assay. (E and F) HCC827 and H3255 cells were transiently transfected with NF- $\mathrm{KB}$ p65 plasmid; 48 hours later, cells were treated with erlotinib for 72 hours, followed by AlamarBlue assay. (C) Overexpression of $\mathrm{p} 65$ was confirmed by Western blot. Western blot results are representative of at least 3 independent replicates. (H) EGFR inhibition promotes expression of NF- $\mathrm{kB}$ target genes. A549 cells were treated with $1 \mu \mathrm{M}$ erlotinib or DMSO for 24 hours. The extracted RNA was subjected to Human NF- $\mathrm{KB}$ Signaling Targets PCR Array (Qiagen). The fold change in levels of NF- $\mathrm{KB}$ target genes is displayed. (I-L) A549 and HCC827 were treated with erlotinib in the presence or absence of NF- $\kappa B$ inhibitor BMS$345541(100 \mathrm{nM})$ or thalidomide $(5 \mu \mathrm{g} / \mathrm{ml})$ for 24 hours. LTB mRNA levels were determined by qPCR. ( $M$ and $\mathbf{N}$ ) A549 and HCC 827 cells were transfected with LTB siRNA for 48 hours, and then treated with erlotinib for 72 hours. Cell viability was measured by AlamarBlue assay. ( $\mathbf{O}$ and $\mathbf{P}$ ) Silencing of LTB was confirmed by qPCR. The erlotinib concentration used was $10 \mathrm{nM}$ for EGFR-mutant cell lines and $1 \mu \mathrm{M}$ for EGFRwt cell lines in $\mathbf{A}-\mathbf{D}$ and $\mathbf{G}-\mathbf{P}$. The erlotinib concentration used was $100 \mathrm{nM}$ in $\mathbf{E}$ and $\mathbf{F}$. For $\mathbf{A}-\mathbf{F}$ and $\mathbf{I}-\mathbf{P}$, data represent the mean \pm SEM. $n=3$ biologically independent experimental replicates. ${ }^{*} P<0.05,{ }^{* *} P<0.01,{ }^{* *} P<0.001$, by Student's $t$ test.

by TNF inhibition. First, we found that TNF was upregulated in all resistant clones compared with the parental cell line (Figure 9C). Next, we found that inhibition of TNF using thalidomide rendered these cells sensitive to EGFR inhibition (Figure 9, D and E, and Supplemental Figure 19, A and B). In addition, we tested H1975 cells (with EGFR L858R/T790M) using afatinib and found that these cells also could be rendered sensitive to EGFR inhibition when TNF was inhibited using thalidomide, etanercept, or siRNA knockdown of TNFR1 (Figure 9F and Supplemental Figure 14, C and D). We examined whether a combined inhibition of EGFR and TNF would be effective in treating H1975 tumors in vivo. H1975 cells were injected into the flanks of mice to form subcutaneous tumors. Once tumors formed, treatment was started. We found that a combined inhibition of afatinib and thalidomide resulted in a highly effective suppression of tumor growth (Figure 9G).

Next, we examined whether a combination of EGFR plus TNF inhibition would prevent the development of secondary resistance in a cell culture model. HCC827 cells were cultured in the continuous presence of erlotinib as described previously (19) in the presence or absence of thalidomide. While prolonged culture in erlotinib alone resulted in the emergence of resistant cells, a combined exposure to erlotinib and thalidomide inhibited the emergence of secondary resistance (Figure $9 \mathrm{H}$ ). A similar effect was found in vivo, where a combination of EGFR plus TNF inhibition prevented the development of acquired EGFR resistance (Figure 9I). Thus, TNF appears to play a role in both intrinsic and acquired resistance to EGFR inhibition, and the effect of TNF can be demonstrated in multiple experimental models.

The TNF adaptive response to EGFR inhibition in NSCLC. Next, we investigated whether activation of $\mathrm{TNF} / \mathrm{NF}-\kappa \mathrm{B}$ signaling can also be detected in tumor tissue derived from patients. We examined 13 TKI-naive and 9 TKI-treated NSCLC patients and found that erlotinib-treated patients had higher TNF levels compared with TKI-naive patients (Figure 10A). We also found increased activation of NF- $\mathrm{KB}$ in erlotinib-treated patients (Figure 10, B and C). Additionally, expression of known NF- $\kappa \mathrm{B}$ target genes was increased in erlotinib-treated lung cancer tissue (Figure 10, D and E). Next, we examined the potential association of TNF and $\mathrm{NF}-\kappa \mathrm{B}$ activation in mitigating EGFR TKI responses in lung cancer patients by analyzing Biomarker-Integrated Approaches of Targeted Therapy for Lung Cancer Elimination (BATTLE) trial data (51). Pre-erlotinib-treatment gene expression data were available from 28 patients who went on to be treated with erlotinib. Three patients within this group had a very short progression-free survival ( $<0.5$ months) and overall survival ( $<1$ month) after starting erlotinib and were excluded from the analysis, since they presumably relapsed before erlotinib could exert its effect. We first looked at whether the presence of TNF or other known NF-kB target genes would mitigate TKI response in patients. Indeed, we find that high levels of TNF and of multiple NF- $\kappa \mathrm{B}$-induced genes $(52,53)$ resulted in a shorter progression-free survival in patients treated with erlotinib in this trial, as determined by the log-rank test. Multiple genes correlated with a shorter progression-free survival, including TNF, IL2RA, LTB, PDGFB, TNFRSF1B (TNFR2), IL12B, CCL22, and TRAF2 (Figure 10F). Since our hypothesis is that resistance to EGFR inhibition is mediated by activation of $\mathrm{NF}-\kappa \mathrm{B}$, we also confirmed that all of these genes were induced by experimental EGFR inhibition in lung cancer cells (Figure 7H). The NF- $\kappa$ B target genes above showed no significant differences between low and high subgroups in sorafenib treatment groups, suggesting a specific effect of EGFR inhibition (Supplemental Figures 20). These data support our model that TNF/NF- $\mathrm{BB}$ activation plays a role in resistance to EGFR inhibition in NSCLC.

\section{Discussion}

The major finding of this study is the identification of an early and widespread mechanism that mediates primary resistance to EGFR inhibition in lung cancer cells, regardless of whether EGFR is wild type or mutant. NSCLC cells respond to EGFR inhibition with a rapid increase in TNF levels, and the TNF upregulation was detected in all NSCLC cell lines examined, in animal tumors derived from NSCLC cell lines, in a direct xenograft model, and in archival tissue from patients. In the case of EGFRwtexpressing NSCLCs, the increase in TNF appears sufficient to protect cells from loss of EGFR signaling. The TNF-driven adaptive response is also detected in NSCLCs with EGFR activating mutations but is only partially protective and is observed at low concentrations of EGFR inhibitors. STAT3 is also rapidly activated upon EGFR inhibition in NSCLCs with EGFR activating mutations (14) and does not seem to inhibit the clinical response in patients. Thus EGFR inhibition in oncogene-addicted cells in the clinical setting may trigger adaptive responses that are ineffective or partially effective. Interestingly, a biologically significant TNF upregulation can also be detected in cells harboring the T790M mutation, suggesting a role in secondary resistance also. Importantly, exogenous TNF protects EGFR-mutant cells from cell death resulting from EGFR inhibition.

EGFR expression is common in NSCLC, and intermediate or high levels of EGFR have been detected in $57 \%-62 \%$ of NSCLCs by immunohistochemistry $(54,55)$. EGFR mutations are detected in $10 \%-15 \%$ of patients of European descent. In general, patients with EGFRwt do not respond to treatment with EGFR TKIs. Although most patients with EGFR activating mutations initial- 
A

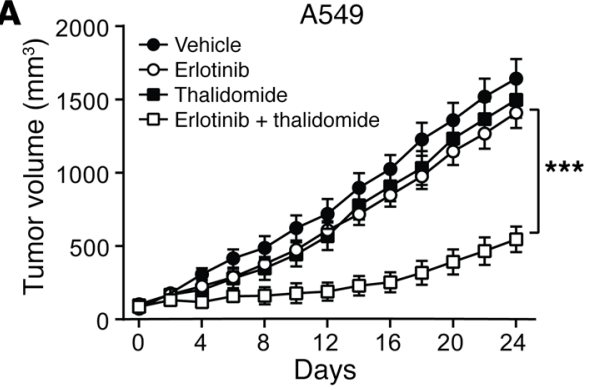

B

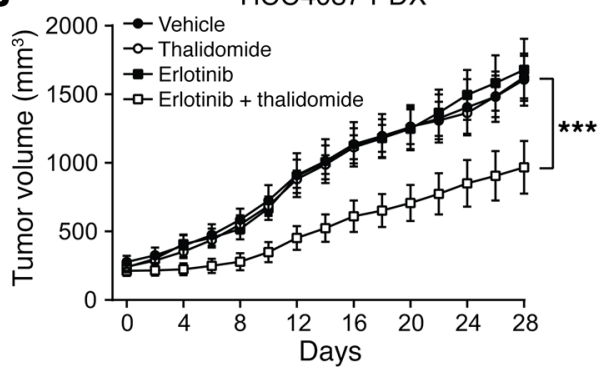

C

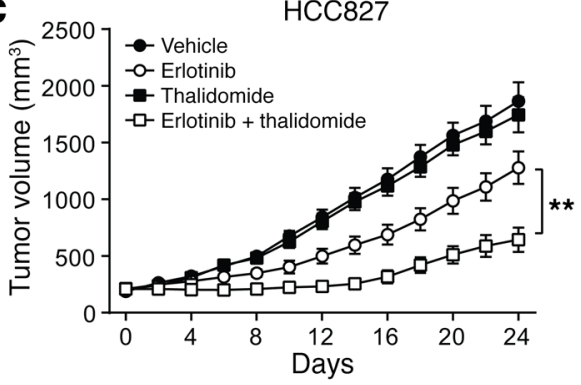

D

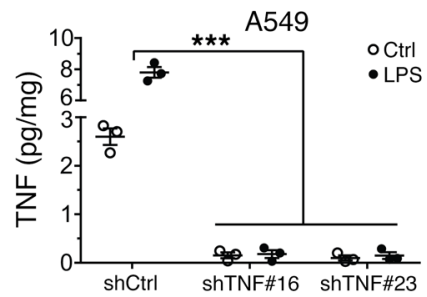

E

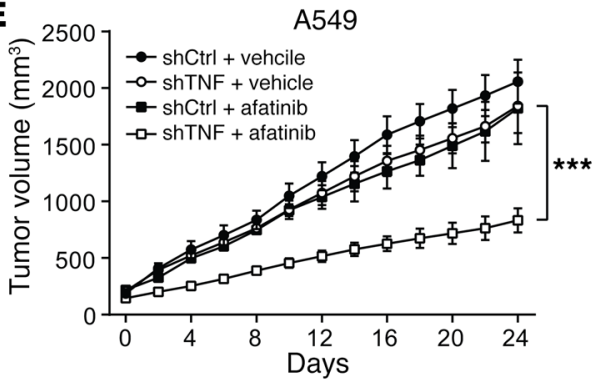

$\mathbf{F}$

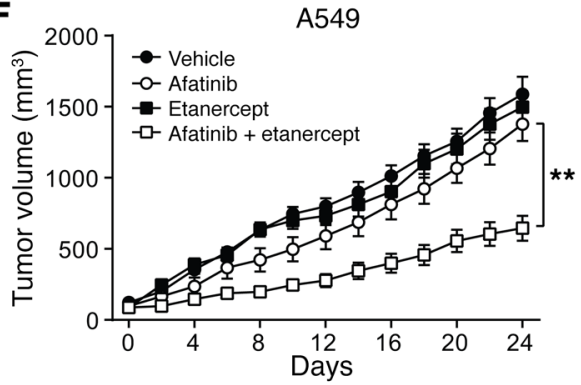

Vehicle

Erlotinib

Thalidomide

Erlotinib + thalidomide
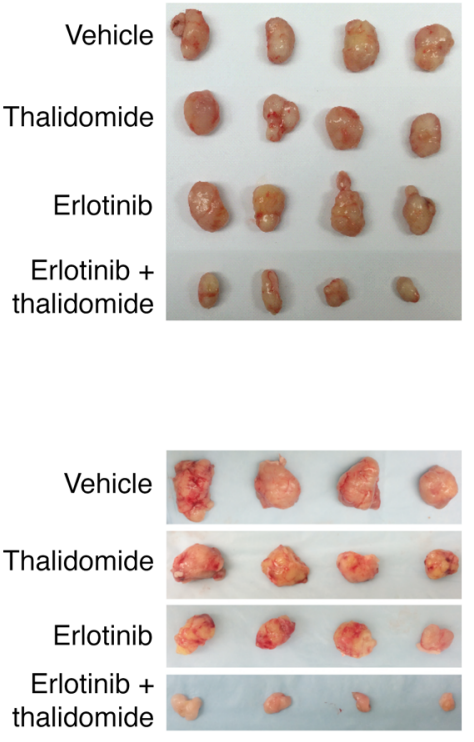

thalidomide
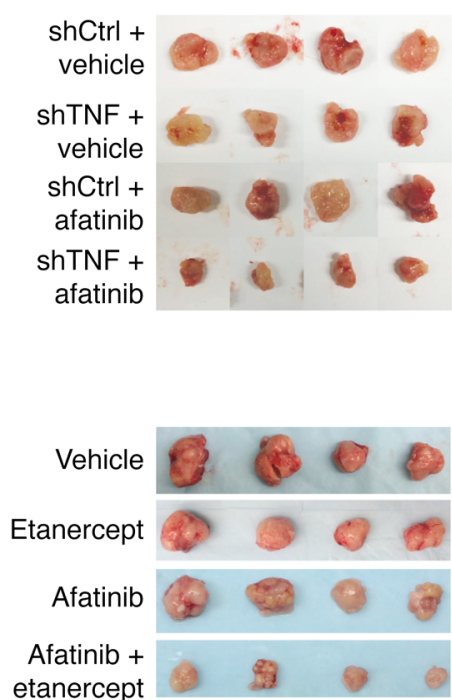

Figure 8. Combined inhibition of EGFR and TNF in mouse models. (A) Athymic mice were injected s.c. with $1 \times 10^{6} \mathrm{~A} 549$ cells. When tumors formed, mice were randomly divided into 4 groups (control, erlotinib, thalidomide, and erlotinib plus thalidomide, $n=8$ ). The mice were treated with $100 \mathrm{mg} / \mathrm{kg}$ erlotinib by oral gavage and/or i.p. injection of $150 \mathrm{mg} / \mathrm{kg}$ thalidomide for 10 consecutive days. (B) HCC4087 EGFRwt NSCLC PDX was implanted s.c. into NOD/SCID mice. When tumors formed, mice were divided into 4 groups $(n=12)$ and treated with $100 \mathrm{mg} / \mathrm{kg}$ erlotinib or $150 \mathrm{mg} /$ $\mathrm{kg}$ thalidomide for 28 days. (C) This experiment was conducted with HCC827 cells $(n=8)$, and mice were treated with erlotinib (10 mg/kg/day) and/ or thalidomide ( $150 \mathrm{mg} / \mathrm{kg} /$ day). (D) Two A549 clones with stable TNF silencing were identified and have low basal and LPS-induced TNF (\#16 and \#23). $n=3$ biologically independent experimental replicates. ${ }^{* * *} P<0.001,2$-sample $t$ test. (E) A549 cells with stably silenced TNF (clone 16) or control shRNA were implanted in flanks of athymic mice. When tumors formed, mice were grouped into control shRNA, TNF shRNA, control shRNA + afatinib, and TNF shRNA + afatinib $(n=6)$. Afatinib $(25 \mathrm{mg} / \mathrm{kg}$ ) was provided by oral gavage. (F) Athymic mice were injected s.c. with A549 cells. When tumors formed, mice were divided into 4 groups (control, afatinib, thalidomide, afatinib plus etanercept, $n=6$ ). The mice were treated by oral gavage of $25 \mathrm{mg} / \mathrm{kg}$ afatinib and/or $3 \mathrm{mg} /$ kg etanercept i.p. Each data point represents the mean tumor volume \pm SEM. Statistical significance was defined as $P<0.05$ (repeatedmeasures 2-way ANOVA, Bonferroni correction for adjusting multiple comparisons, between EGFR inhibition group and combination of EGFR and TNF inhibition group, by GraphPad Prism 7.0). ${ }^{* *} P<0.01,{ }^{* *} P<0.001$. 
A

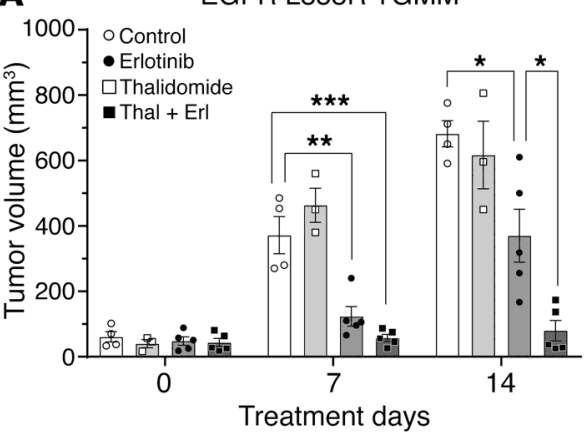

B

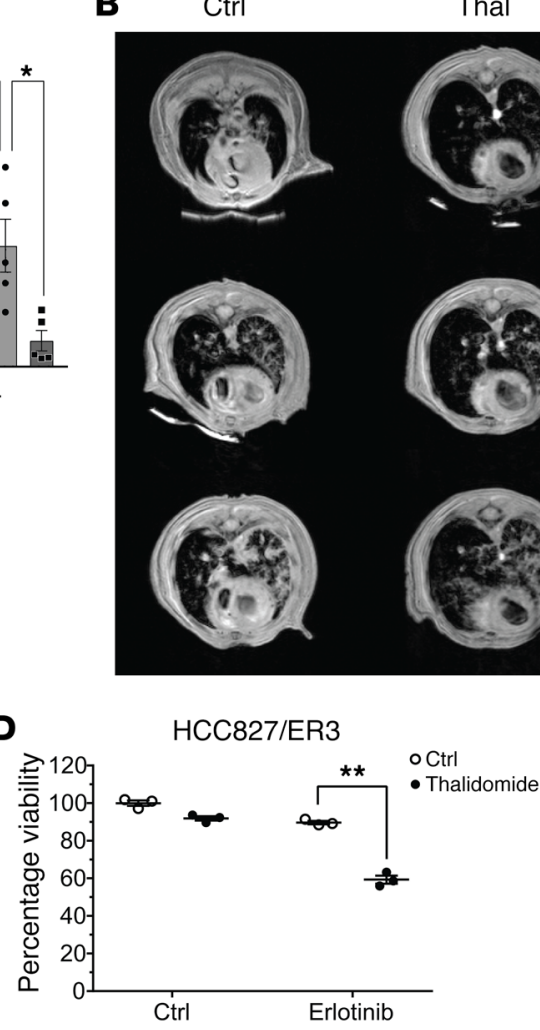

D

H1975

G
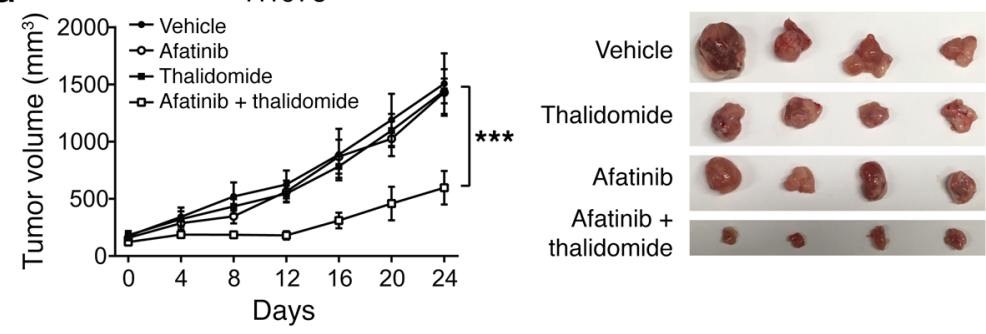

E

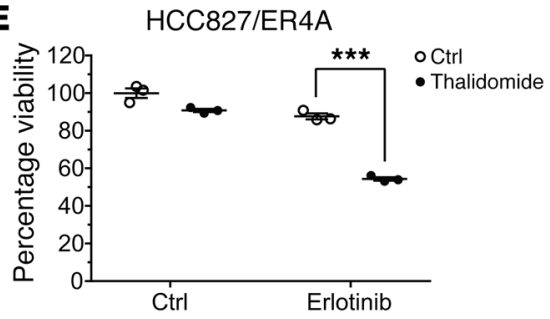

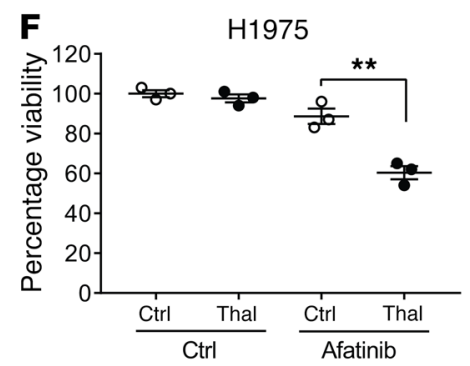
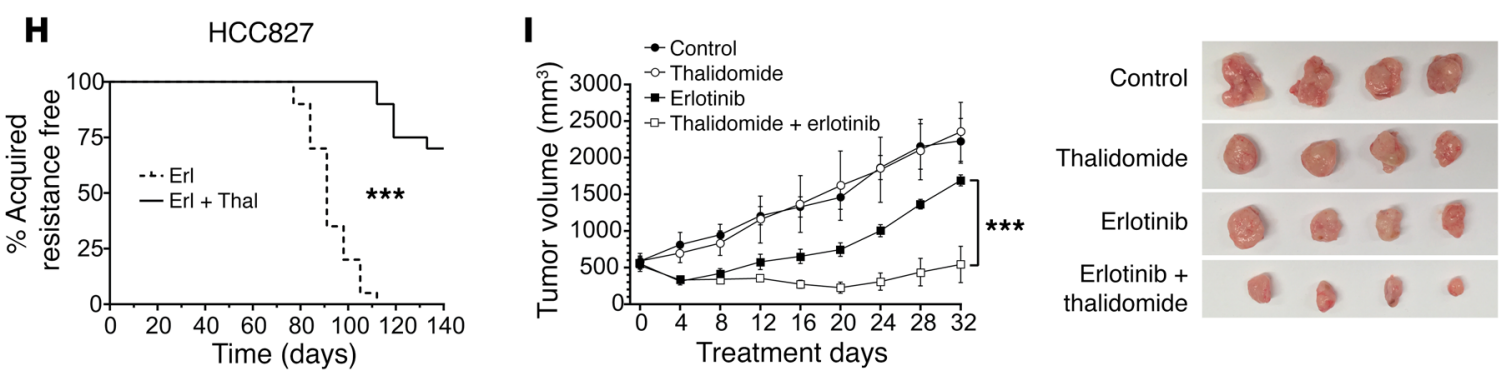

Figure 9. EGFR and TNF inhibition prevents the acquired resistance. (A) TetO-EGFR-L858R and CCSP-rtTA mice were exposed to doxycycline diets to induce tumors. Lung tumor formation was confirmed by MRI between weeks 4 and 5 . Starting from week 5 , mice were randomly divided into 4 groups and treated by $6.25 \mathrm{mg} / \mathrm{kg}$ erlotinib and/or $150 \mathrm{mg} / \mathrm{kg}$ thalidomide for 2 weeks. Tumor sizes were measured by 2 blinded researchers using Image). Data were presented as each tumor size and the mean \pm SEM. (B) MRI images from representative mice in each group. The tumors grow as diffuse lung opacities. (C) TNF mRNAs were detected in HCC827 parent and erlotinib-resistant cell lines by qPCR. (D-F) HCC827/ER3, HCC827/ER4A, and H1975 cells were treated with $5 \mu \mathrm{g} / \mathrm{ml}$ thalidomide and/or $100 \mathrm{nM}$ erlotinib/afatinib for 72 hours followed by AlamarBlue assay. (C) $1 \times 10^{6} \mathrm{H} 1975$ cells were injected s.c. into athymic mice. When tumors formed, mice were divided into 4 groups $(n=6)$ and treated with $5 \mathrm{mg} / \mathrm{kg}$ afatinib by oral gavage or $150 \mathrm{mg} / \mathrm{kg}$ thalidomide i.p. for 24 days. (H) HCC827 cells were planted in a 96-well plate and treated with $100 \mathrm{nM}$ erlotinib with or without $5 \mu \mathrm{g} / \mathrm{ml}$ thalidomide. The day when cells reached $100 \%$ confluence was considered the appearance of acquired resistance. (I) HCC827 cells were injected subcutaneously into athymic mice. When tumors reached $500 \mathrm{~mm}^{3}$, mice were divided into 4 groups $(n=6)$ and treated with $6.25 \mathrm{mg} / \mathrm{kg}$ erlotinib by oral gavage or $150 \mathrm{mg} / \mathrm{kg}$ thalidomide by i.p. for 32 days. For (C-F) data represent mean \pm SEM. $n=3$ biologically independent experimental replicates. ${ }^{*} P<0.05,{ }^{* *} P<0.01,{ }^{* * *} P<0.001$, by 2 -sample $t$-test, except $\mathbf{H}$ by logrank test. For $\mathbf{G}$ and $\mathbf{I}$, statistical significance was defined as $P<0.05$ (repeated-measures 2-way ANOVA, Bonferroni correction, GraphPad Prism 7.0). 
A

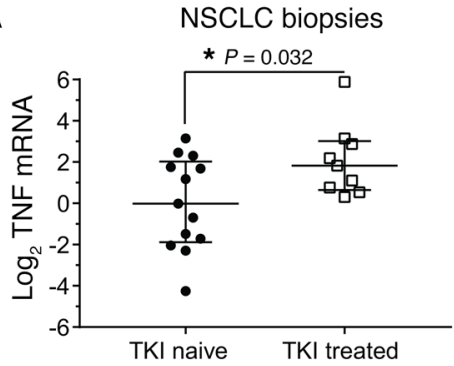

B

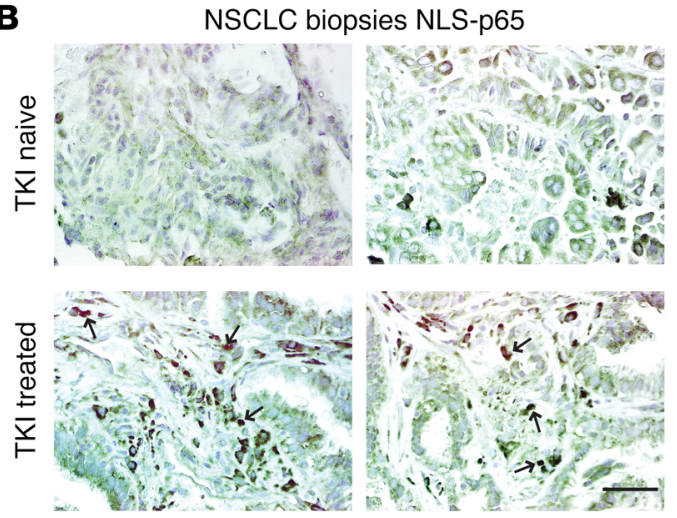

C

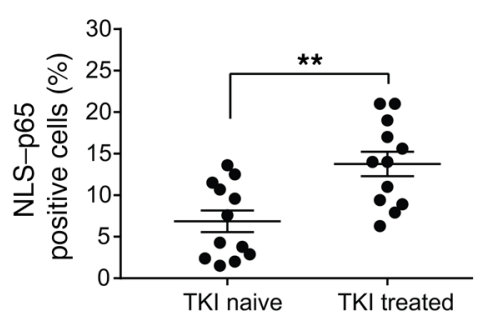

F

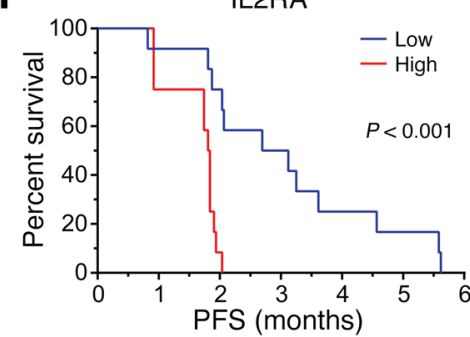

TNF
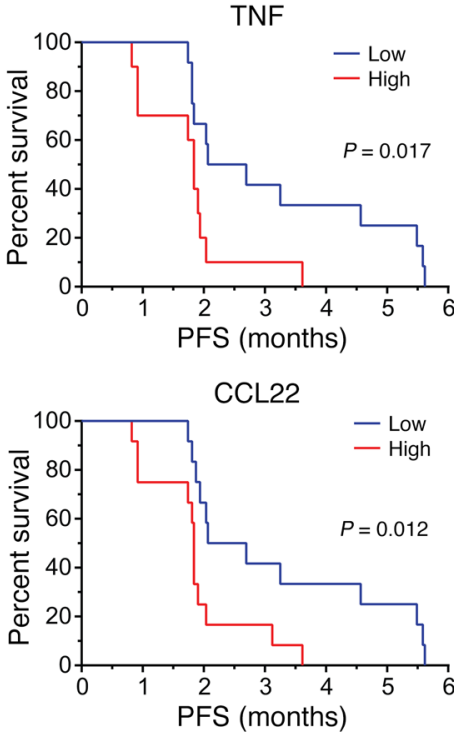

D

NSCLC biopsies

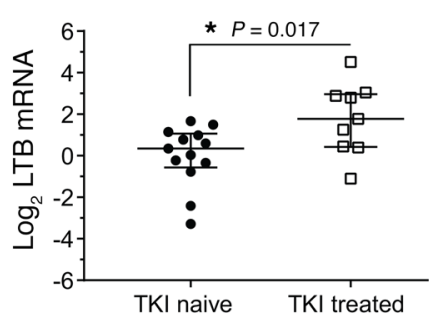

LTB

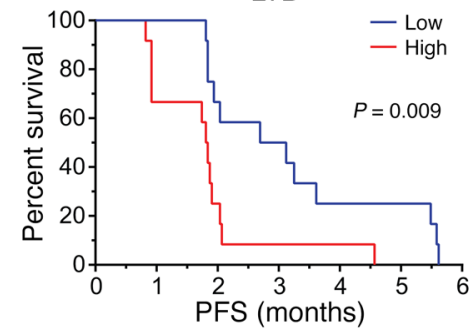

TNFRSF1B (TNFR2)

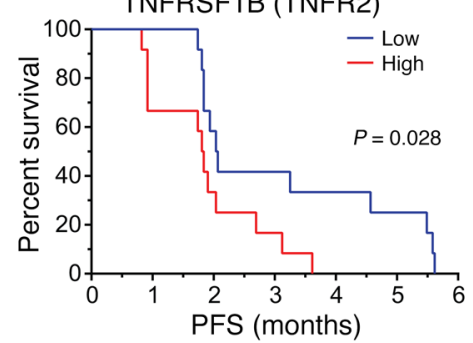

TRAF2

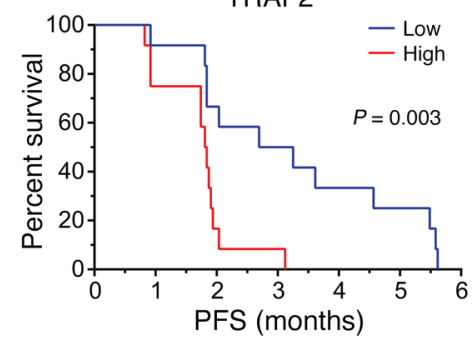

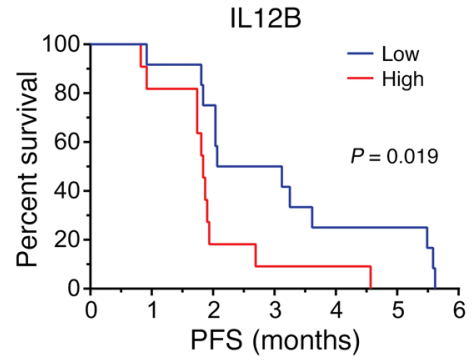

E NSCLC biopsies

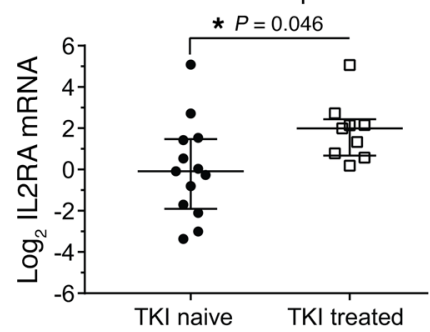

PDGFB

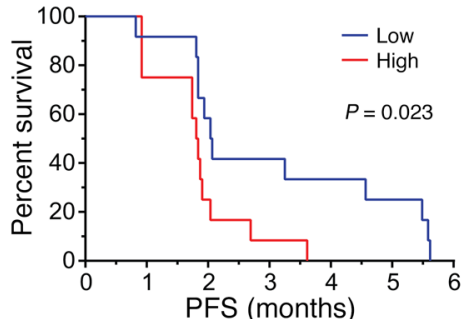

IL12B

Figure 10. Erlotinib induces TNF and p65 activation in patient tissue. (A, D, and E) RNA was extracted from FFPE tissue from 22 NSCLC patients (13 untreated and 9 treated with erlotinib). TNF, LTB, and IL2RA mRNA levels were quantified by qPCR. Each dot represents the log $\mathrm{g}_{2} \mathrm{mNA}$ level of each patient and median \pm interquartile range. ${ }^{*} P<0.05$, by Student's $t$ test. (B) Immunostaining of nuclear-localized NF- $\kappa B$ p 65 from representative tumor sections of 2 different patients in TKI-untreated (top) and -treated groups (bottom) $(n=3)$. p65-positive cells are indicated by black arrows. Scale bar: $50 \mu \mathrm{m}$. (C) Quantification of p65-positive cells. Four fields $(\times 400)$ were randomly selected for each tissue block $(n=12)$. Data represent the mean \pm SEM. ${ }^{* *} P<0.01$, by Student's $t$ test. $(\mathbf{F})$ Association between patients' progression-free survival (PFS) and their TNF/NF- $\kappa$ B gene expression profiles in the BATTLE trial was analyzed by log-rank test. Low or high levels of gene were defined as higher or lower than the median value of 25 patients. $P$ less than 0.05 was considered statistically significant. 
ly respond to EGFR TKIs, they inevitably developed a secondary resistance to this treatment, implying the persistence of subsets of cancer cells that are not eliminated during the initial treatment. Thus, a more effective elimination of cancer cells during the initial treatment may delay or abrogate the emergence of secondary resistance. In addition, it may be possible to overcome the secondary resistance of NSCLC with appropriately targeted treatments. Our studies indicate that combined inhibition of EGFR and TNF may prevent the development of secondary resistance. Furthermore, NSCLC cells with secondary resistance to EGFR inhibition become sensitive to EGFR TKIs if combined with TNF inhibition.

Primary or intrinsic resistance to EGFRwt inhibition could occur because the EGFRwt does not drive the survival/proliferation of these cells. Alternatively, an adaptive response could prevent cell death in response to EGFR inhibition. Currently the EGFRwt does not appear to be a useful target for treatment, because EGFR inhibition is ineffective in EGFRwt-expressing NSCLC. However, EGFRwt overexpression is common, and the presence of EGFR ligand is common and well documented in lung cancer $(56,57)$. Furthermore, a constitutive overexpressioninduced EGFRwt signaling has also been reported (58-60). Thus, it seems likely that EGFRwt is active in lung cancer. Our data indicate that if the TNF-driven adaptive response is inhibited, EGFRwtexpressing NSCLCs become sensitive to EGFR inhibition.

We propose that EGFR inhibition results in an increase in TNF levels via a dual mechanism (as shown in the schematic in Supplemental Figure 21). First, we demonstrate that activation of EGFR signaling results in a rapid downregulation of TNF mRNA by a rapid induction of miR-21, and we find that inhibition of EGFR results in increased TNF mRNA stability by a decrease in miR-21 levels. A second mechanism involves the transcription factor $N F-\kappa B$. TNF activates NF- $\kappa$, which in turn increases the transcription of TNF mRNA in a feed-forward loop. Our data suggest that TNF-mediated activation of $\mathrm{NF}-\kappa \mathrm{B}$ is likely to be a major mechanism of resistance to EGFR inhibition. Downstream of NF- $\kappa \mathrm{B}$ we identify lymphotoxin- $\beta$ as a key mediator of resistance to EGFR inhibition.

The biological effect of increased TNF signaling is protection from cell death mediated by a loss of EGFR signaling. We examined the combined effect of TNF and EGFR inhibition in a resistant EGFRwt cell line, A549 cells, in a mouse model using multiple approaches to inhibit TNF. A combination of EGFR TKI plus thalidomide was also highly effective in inhibiting tumor growth in an EGFRwt PDX model, while EGFR inhibition or thalidomide alone was ineffective. Thalidomide is a known inhibitor of TNF and may regulate TNF transcription and/or stability $(37,38)$. Using a low concentration of erlotinib, we also noted a significant reduction in tumor growth with a combined inhibition of TNF and EGFR in HCC827 cells compared with EGFR inhibition alone, although the tumors were sensitive to EGFR inhibition alone. Importantly, a similar approach of combining EGFR plus TNF inhibition is also effective in an immunocompetent transgenic EGFR-mutant model, suggesting that this approach may be therapeutically useful in NSCLC patients.

NSCLC is a common cancer worldwide, constituting about $85 \%$ of all lung cancer. A biologically significant upregulation of TNF upon EGFR inhibition may have enormous implications for the treatment of lung cancer and could greatly expand the reach and impact of EGFR-targeted treatment in NSCLC. A majority of NSCLCs express EGFRwt, with a smaller subset expressing EGFR activating mutations. The therapeutic approach we propose is potentially applicable to the majority of NSCLCs, including EGFRwt-expressing cancers and including the subset with EGFR activating mutations. Our findings suggest that inhibiting EGFR with a combination of TKI plus a TNF inhibitor such as thalidomide or etanercept may be effective in the treatment of NSCLCs that express EGFRwt. In tumors with EGFR activating mutations, a combined treatment with EGFR and TNF inhibition may result in a more effective elimination of tumor cells during the initial treatment and perhaps eliminate or delay secondary resistance. We also found TNF upregulation in H1975 cells (harboring T790M mutation), and found that combined TNF and EGFR inhibition is effective in a mouse model, hinting that this approach may be worth testing in tumors with secondary resistance. We have recently shown that EGFR inhibition triggers a biologically significant TNF upregulation that mediates primary resistance to EGFR inhibition in glioblastoma (GBM). Like in lung cancer, the TNF upregulation in GBM is also detected in EGFRwt- or EGFR mutant-expressing glioma cells. However, our initial studies indicate that the EGFR inhibition-triggered signaling networks downstream of TNF are distinct in brain and lung cancers. In GBM, TNF activates a JNK/Axl/ERK signaling axis (40, 61), while in lung cancer, the major downstream effector is NF- $\kappa \mathrm{B}$. EGFR expression is widespread in other types of human cancer $(62,63)$, and it will be interesting to determine whether a biologically significant upregulation of TNF in response to EGFR inhibition is a widespread feature of human cancers of epithelial origin.

\section{Methods}

\section{Plasmids, transfection, and generation of cell lines}

Calu-3 and A549 cells were obtained from American Type Culture Collection (ATCC). HCC827/ER3 and HCC827/ER4(A) were generated as described previously (11). HCC827/ER4(B) and HCC827/T790M were obtained from Eric Haura, Moffitt Cancer Center (Tampa, Florida, USA) (50). All other cell lines were obtained from the Hamon Center for Therapeutic Oncology Research at the University of Texas Southwestern Medical Center (UT Southwestern) (and deposited at the ATCC). Cells were cultured in RPMI-1640 in 5\% FBS for all experiments except those involving the use of EGF. In all experiments involving use of EGF, cells were cultured overnight in serum-free RPMI-1640, and EGF was added to serum-free medium. In such experiments, cells not treated with EGF were also serum-starved. Cell lines were DNA fingerprinted using Promega StemElite ID system, a short tandem repeat-based assay, at UT Southwestern genomics core and mycoplasma tested using an e-Myco kit (Boca Scientific). p65 expression plasmid was obtained from Stratagene. NF- $\mathrm{kB}-\mathrm{LUC}$ plasmid was provided by Ezra Burstein (UT Southwestern). TNF 3'-UTR luciferase reporter (SV40-luc-TNF) was a gift from Jay Steer and David Joyce, University of Western Australia (Perth, Western Australia, Australia).

\section{RNA interference}

For transient silencing we used a pool of siRNA sequences directed against human TNFR1, LTB, or control (scrambled) siRNA, all 
obtained from Santa Cruz Biotechnology. siRNA knockdown was performed according to the manufacturer's protocol using Lipofectamine 2000 reagent (Invitrogen). Experiments were conducted 48 hours after siRNA transfection.

\section{Antibodies, reagents, and Western blotting}

Western blot and immunoprecipitation were performed according to standard protocols. Western blots are representative of at least 3 independent experiments. A list of antibodies used is provided in Supplemental Methods. Uncropped Western blots are displayed in Supplemental Figures 22-24.

Recombinant human TNF and EGF were obtained from PeproTech. Erlotinib was purchased from SelleckChem. Afatinib was bought from AstaTech Inc. Thalidomide, actinomycin D, and mithramycin A were from Cayman Chemical. Etanercept (Enbrel) was purchased from McKesson Medical Supply. The NF-кB inhibitors BMS-345541, QNZ (EVP 4593), and sodium salicylate were obtained from MilliporeSigma.

\section{cDNA synthesis and real-time PCR}

Total RNA was isolated by TRIzol Reagent (Fisher Scientific). cDNA reverse transcription was performed using a High-Capacity cDNA Reverse Transcription Kit (Applied Biosystems). PCR primers were synthesized by Integrated DNA Technologies Inc. (IDT). Each PCR reaction was carried out in triplicate in a $20-\mu$ l volume using SYBR Green Master Mix (Applied Biosystems). Additional details and primer sequences are provided in Supplemental Methods.

\section{MicroRNA studies}

For microRNA quantitation, we used a mirVana miRNA Isolation Kit (Ambion) to isolate the high-quality small RNAs. TaqMan MicroRNA Reverse Transcription Kit (Applied Biosystems) was used for converting microRNA to cDNA. The RT primers were within the TaqMan MicroRNA Assay hsa-miR-21-5p and hsa-miR-423-5p (Thermo Fisher Scientific). hsa-miR-423-5p was used as the endogenous control. PCR reactions were performed in triplicate by TaqMan Universal Master Mix II (Applied Biosystems), using the same PCR program as SYBR Green Master Mix. PCR primers of hsa-miR-21-5p and hsa-miR-423$5 p$ were from TaqMan MicroRNA Assay (Thermo Fisher Scientific). Each experiment was carried out independently at least 3 times. The miR-21 expression levels were normalized to miR-423. Additional details of microRNA inhibition and microRNA overexpression are provided in Supplemental Methods.

\section{Cell viability assay}

Cell viability assay was conducted using AlamarBlue cell viability assay from Thermo Fisher Scientific, according to the manufacturer's protocol. Cells were treated by the indicated drugs for 72 hours before detection. In AlamarBlue cell viability assay, cells were cultured in Corning 96-well black plates with clear bottom, and the detection was carried out under the fluorimeter (excitation at $544 \mathrm{~nm}$ and emission at $590 \mathrm{~nm}$ ) using a POLARstar Omega Microplate Reader (BMG LABTECH). At least 3 independent experiments were done.

\section{Animal studies}

Four- to six-week-old female athymic mice were purchased from Charles River Laboratories. A549 cells $\left(1 \times 10^{6}\right)$, HCC827 cells $\left(2 \times 10^{6}\right)$, or H1975 cells $\left(1 \times 10^{6}\right)$ were injected s.c. into the flanks of athymic mice.
About 10 days after injection, all mice had developed subcutaneous tumors. The mice were randomly divided into indicated groups. Mice were treated with drugs using the doses described in the figure legends. For combination treatment, both drugs were given concurrently for indicated periods. Tumor dimensions were measured every 2 days and tumor volumes calculated by the formula: volume $=($ length $\times$ width $\times$ width) $/ 2$. Mice were sacrificed when tumors reached over $2,000 \mathrm{~mm}^{3}$ or after the indicated days. HCC4087 PDX model was established at UT Southwestern as described in Supplemental Methods.

Transgenic mouse model. Four- to six-week-old female and male TetO-EGFR-L858R and CCSP-rtTA mice were generated in Katerina Politi's laboratory at Yale University (New Haven, Connecticut, USA) and shipped to UT Southwestern for further experiments. Mice were given continuous doxycycline in diet (TD.01306 2018625 Doxycycline, Envigo) to induce tumors. Lung tumor formation was confirmed by comparison of MRI between 4 and 5 weeks of doxycycline induction. Starting from week 5 , mice were randomly divided into indicated groups and treated for 2 weeks. Tumor sizes were measured from MRI images by 2 blinded researchers using Image (NIH).

MRI imaging. MRI was done at the Mouse MRI Core, Advanced Imaging Research Center, at UT Southwestern. Additional details regarding MRI studies are provided in Supplemental Methods.

\section{Patient specimens}

Twenty-two NSCLC patients' formalin-fixed, paraffin-embedded (FFPE) tissues were obtained from The Jackson Laboratory or UT Southwestern according to IRB-approved protocols. Thirteen specimens were obtained from UT Southwestern and 9 from The Jackson Laboratory. Thirteen patients had no EGFR TKI treatment, and 9 patients had undergone EGFR TKI treatment.

BATTLE trial data (ClinicalTrials.gov NCT00410059 for erlotinib, NCT00411671 for sorafenib) were provided by the BATTLE group at MD Anderson Cancer Center.

\section{Statistics}

Error bars represent the means \pm SEM of 3 independent experiments if not indicated. The subcutaneous tumor growth data were analyzed by repeated-measures 2-way ANOVA. Bonferroni correction was used to adjust the significance level for multiple comparisons, and the family-wise error rate (FWER) was set at 0.05. The significance of differences between 2 groups was determined by the $P$ value of the treatment factor (the significance between EGFR inhibition and combination of EGFR plus TNF inhibition is shown). Kaplan-Meier survival curves were constructed and compared by log-rank test. For comparing multiple groups versus the same control, 1-way ANOVA with Dunnett's method and a FWER of 0.05 was performed to calculate the adjusted $P$ value. All other data were analyzed for significance between the indicated treated group and control group, with 2-tailed 2-sample $t$ test. All analyses above were performed using GraphPad Prism 7.0 software. A $P$ value or an adjusted $P$ value for multiple comparison less than 0.05 was considered statistically significant $\left({ }^{*} P<0.05,{ }^{* *} P<0.01,{ }^{* *} P<0.001,{ }^{* * *} P<0.0001\right.$; ${ }^{*}$ not statistically significant).

\section{Study approval}

All animal studies were done under IACUC-approved protocols at UT Southwestern, North Texas VA Medical Center (Dallas, Texas, USA), 
and Yale University (New Haven, Connecticut, USA). Human tissues were obtained from UT Southwestern after IRB approval and informed consent. The BATTLE trial was registered at US ClinicalTrials.gov.

Additional experimental details are provided in Supplemental Methods.

\section{Author contributions}

$\mathrm{KJH}, \mathrm{HZ}$, and MT analyzed data. AAH, KG, and GG designed experiments. $\mathrm{KG}, \mathrm{GG}, \mathrm{BG}, \mathrm{CH}$, and SZ performed experiments. BG, DEG, MP, CH, JDM, KK, FJF, VAP, VO, and TGB provided resources. LC, YX, BM, SB, DZ, SZ, KD, JD, KG, GG, and AAH analyzed data. AAH, KG, and GG wrote the manuscript with contributions from DEG and JDM. AAH conceived the study and supervised it.

\section{Acknowledgments}

This work was supported in part by NIH grant R01 NS062080 and by the Office of Medical Research at the Department of Veterans Affairs, a Lung Cancer SPORE Career Enhancement Program Award, and support from the Dallas VA Research Corporation to
AAH. This work was also supported by National Cancer Institute Lung Cancer SPORE (P50CA70907), U01CA176284, and the Cancer Prevention Research Institute of Texas (RP110708) to JDM. This work was also supported by NIH grant 1R01CA194578 to DZ and by NIH grants R01CA169338 and U54CA224081 to TGB. DEG is supported by a National Cancer Institute Midcareer Investigator Award in Patient-Oriented Research, K24CA201543-01. SB is supported by grants from the NIH (RO1CA197796, RO1CA149461, and R21CA202403) and the National Aeronautics and Space Administration (NNX16AD78G). We thank Katerina Politi for generating and providing TetO-EGFR-L858R and CCSP-rtTA mice. We thank Jay Steer and David Joyce for TNF-UTR plasmid, Eric Haura for providing erlotinib-resistant cell lines, and Jessica Saltarski (UT Southwestern) for assistance in obtaining FFPE tissues.

Address correspondence to: Amyn A. Habib, University of Texas Southwestern Medical Center, VA North Texas Health Care System, Mail Code 151, 4500 South Lancaster Road, Dallas, Texas 75216, USA. Phone: 214.857.3610; Email: Amyn.Habib@ UTSouthwestern.edu.
1. Sharma SV, Bell DW, Settleman J, Haber DA. Epidermal growth factor receptor mutations in lung cancer. Nat Rev Cancer. 2007;7(3):169-181.

2. Weinstein IB. Cancer. Addiction to oncogenes - the Achilles heal of cancer. Science. 2002;297(5578):63-64.

3. Guo A, et al. Signaling networks assembled by oncogenic EGFR and c-Met. Proc Natl Acad Sci US A. 2008;105(2):692-697.

4. Paez JG, et al. EGFR mutations in lung cancer: correlation with clinical response to gefitinib therapy. Science. 2004;304(5676):1497-1500.

5. Lynch TJ, et al. Activating mutations in the epidermal growth factor receptor underlying responsiveness of non-small-cell lung cancer to gefitinib. N Engl JMed. 2004;350(21):2129-2139.

6. Pao W, et al. EGF receptor gene mutations are common in lung cancers from "never smokers" and are associated with sensitivity of tumors to gefitinib and erlotinib. Proc Natl Acad Sci U S A. 2004;101(36):13306-13311.

7. Dowell JE, Minna JD. EGFR mutations and molecularly targeted therapy: a new era in the treatment of lung cancer. Nat Clin Pract Oncol. 2006;3(4):170-171.

8. Chong CR, Jänne PA. The quest to overcome resistance to EGFR-targeted therapies in cancer. Nat Med. 2013;19(11):1389-1400.

9. Yu HA, et al. Analysis of tumor specimens at the time of acquired resistance to EGFR-TKI therapy in 155 patients with EGFR-mutant lung cancers. Clin Cancer Res. 2013;19(8):2240-2247.

10. Engelman JA, et al. MET amplification leads to gefitinib resistance in lung cancer by activating ERBB3 signaling. Science. 2007;316(5827):1039-1043.

11. Zhang Z, et al. Activation of the AXL kinase causes resistance to EGFR-targeted therapy in lung cancer. Nat Genet. 2012;44(8):852-860.

12. Rotow J, Bivona TG. Understanding and targeting resistance mechanisms in NSCLC. Nat Rev Cancer. 2017;17(11):637-658.

13. Fan W, et al. MET-independent lung cancer cells evading EGFR kinase inhibitors are therapeutically susceptible to BH3 mimetic agents. Cancer Res. 2011;71(13):4494-4505.

14. Lee HJ, Zhuang G, Cao Y, Du P, Kim HJ, Settleman J. Drug resistance via feedback activation of Stat 3 in oncogene-addicted cancer cells. Cancer Cell. 2014;26(2):207-221.

15. Liao S, Davoli T, Leng Y, Li MZ, Xu Q, Elledge SJ. A genetic interaction analysis identifies cancer drivers that modify EGFR dependency. Genes Dev. 2017;31(2):184-196.

16. Hata AN, et al. Tumor cells can follow distinct evolutionary paths to become resistant to epidermal growth factor receptor inhibition. Nat Med. 2016;22(3):262-269.

17. Ohashi K, Maruvka YE, Michor F, Pao W. Epidermal growth factor receptor tyrosine kinase inhibitor-resistant disease. J Clin Oncol. 2013;31(8):1070-1080.

18. Greulich $\mathrm{H}$, et al. Oncogenic transformation by inhibitor-sensitive and -resistant EGFR mutants. PLoS Med. 2005;2(11):e313.

19. Blakely CM, et al. NF-кB-activating complex engaged in response to EGFR oncogene inhibition drives tumor cell survival and residual disease in lung cancer. Cell Rep. 2015;11(1):98-110.

20. Yun $\mathrm{CH}$, et al. Structures of lung cancerderived EGFR mutants and inhibitor complexes: mechanism of activation and insights into differential inhibitor sensitivity. Cancer Cell. 2007;11(3):217-227.

21. Mulloy R, et al. Epidermal growth factor receptor mutants from human lung cancers exhibit enhanced catalytic activity and increased sensitivity to gefitinib. Cancer Res. 2007;67(5):2325-2330.

22. Bartel DP. MicroRNAs: target recognition and regulatory functions. Cell. 2009;136(2):215-233.

23. Lin S, Gregory RI. MicroRNA biogenesis pathways in cancer. Nat Rev Cancer. 2015;15(6):321-333.

24. Lu J, et al. MicroRNA expression profiles classify human cancers. Nature. 2005;435(7043):834-838.

25. Yanaihara N, et al. Unique microRNA molecular profiles in lung cancer diagnosis and prognosis. Cancer Cell. 2006;9(3):189-198.

26. Seike M, et al. MiR-21 is an EGFR-regulated anti-apoptotic factor in lung cancer in never-smokers. Proc Natl Acad Sci U S A. 2009;106(29):12085-12090.

27. Bivona TG, et al. FAS and NF- $\kappa \mathrm{B}$ signalling modulate dependence of lung cancers on mutant EGFR. Nature. 2011;471(7339):523-526.

28. Wajant H, Pfizenmaier K, Scheurich P. Tumor necrosis factor signaling. Cell Death Differ. 2003;10(1):45-65.

29. Zhang X, et al. MicroRNA-21 modulates the levels of reactive oxygen species by targeting SOD3 and TNF $\alpha$. Cancer Res. 2012;72(18):4707-4713.

30. Barnett RE, et al. Anti-inflammatory effects of miR-21 in the macrophage response to peritonitis. J Leukoc Biol. 2016;99(2):361-371.

31. Das A, Ganesh K, Khanna S, Sen CK, Roy S. Engulfment of apoptotic cells by macrophages: a role of microRNA-21 in the resolution of wound inflammation. J Immunol. 2014;192(3):1120-1129.

32. Fabian MR, Sonenberg N, Filipowicz W. Regulation of mRNA translation and stability by microRNAs. Annu Rev Biochem. 2010;79:351-379.

33. Park PH, Huang H, McMullen MR, Mandal P, Sun L, Nagy LE. Suppression of lipopolysaccharide-stimulated tumor necrosis factor-alpha production by adiponectin is mediated by transcriptional and post-transcriptional mechanisms. JBiol Chem. 2008;283(40):26850-26858.

34. Zhu J, et al. TNF- $\alpha$ mRNA is negatively regulated by microRNA-181a-5p in maturation of dendritic cells induced by high mobility group box-1 protein. Sci Rep. 2017;7(1):12239.

35. Brenner D, Blaser H, Mak TW. Regulation of tumour necrosis factor signalling: live or let die. Nat Rev Immunol. 2015;15(6):362-374.

36. Faustman D, Davis M. TNF receptor 2 pathway: drug target for autoimmune diseases. Nat Rev Drug Discov. 2010;9(6):482-493.

37. Sedger LM, McDermott MF. TNF and TNF- 
receptors: from mediators of cell death and inflammation to therapeutic giants - past, present and future. Cytokine Growth Factor Rev. 2014;25(4):453-472.

38. Moreira AL, Sampaio EP, Zmuidzinas A, Frindt P, Smith KA, Kaplan G. Thalidomide exerts its inhibitory action on tumor necrosis factor alpha by enhancing mRNA degradation. JExp Med. 1993;177(6):1675-1680.

39. Keifer JA, Guttridge DC, Ashburner BP, Baldwin AS. Inhibition of NF-kappa B activity by thalidomide through suppression of IkappaB kinase activity. J Biol Chem. 2001;276(25):22382-22387.

40. Guo G, et al. A TNF-JNK-Axl-ERK signaling axis mediates primary resistance to EGFR inhibition in glioblastoma. Nat Neurosci. 2017;20(8):1074-1084.

41. Ichihara $\mathrm{E}$, et al. SFK/FAK signaling attenuates osimertinib efficacy in both drug-sensitive and drug-resistant models of EGFR-mutant lung cancer. Cancer Res. 2017;77(11):2990-3000.

42. Lee JE, et al. Hippo pathway effector YAP inhibition restores the sensitivity of EGFR-TKI in lung adenocarcinoma having primary or acquired EGFR-TKI resistance. Biochem Biophys Res Commun. 2016;474(1):154-160.

43. Chaib I, et al. Co-activation of STAT3 and YES-associated Protein 1 (YAP1) pathway in EGFR-mutant NSCLC. J Natl Cancer Inst. 2017;109(9):djx014.

44. Balkwill $\mathrm{F}$. Tumour necrosis factor and cancer. Nat Rev Cancer. 2009;9(5):361-371.

45. Sethi G, Sung B, Aggarwal BB. TNF: a master switch for inflammation to cancer. Front Biosci. 2008;13:5094-5107.
46. Fernandes MT, Dejardin E, dos Santos NR. Context-dependent roles for lymphotoxin- $\beta$ receptor signaling in cancer development. Biochim Biophys Acta. 2016;1865(2):204-219.

47. Hsu DS, et al. Lymphotoxin- $\beta$ interacts with methylated EGFR to mediate acquired resistance to cetuximab in head and neck cancer. Clin Cancer Res. 2017;23(15):4388-4401.

48. Scarzello AJ, et al. LT $\beta$ R signalling preferentially accelerates oncogenic AKT-initiated liver tumours. Gut. 2016;65(10):1765-1775.

49. Politi K, Zakowski MF, Fan PD, Schonfeld EA, Pao W, Varmus HE. Lung adenocarcinomas induced in mice by mutant EGF receptors found in human lung cancers respond to a tyrosine kinase inhibitor or to down-regulation of the receptors. Genes Dev. 2006;20(11):1496-1510.

50. Yoshida T, et al. Tyrosine phosphoproteomics identifies both codrivers and cotargeting strategies for T790M-related EGFR-TKI resistance in non-small cell lung cancer. Clin Cancer Res. 2014;20(15):4059-4074.

51. Kim ES, et al. The BATTLE trial: personalizing therapy for lung cancer. Cancer Discov. 2011;1(1):44-53.

52. Oeckinghaus A, Ghosh S. The NF- $\mathrm{BB}$ family of transcription factors and its regulation. Cold Spring Harb Perspect Biol. 2009;1(4):a000034.

53. Karin M, Cao Y, Greten FR, Li ZW. NF-кB in cancer: from innocent bystander to major culprit. Nat Rev Cancer. 2002;2(4):301-310.

54. Tsao MS, et al. Erlotinib in lung cancer - molecular and clinical predictors of outcome. $N$ EnglJ
Med. 2005;353(2):133-144.

55 . Hirsch FR, et al. Epidermal growth factor receptor in non-small-cell lung carcinomas: correlation between gene copy number and protein expression and impact on prognosis. J Clin Oncol. 2003;21(20):3798-3807.

56. Hsieh ET, Shepherd FA, Tsao MS. Co-expression of epidermal growth factor receptor and transforming growth factor- $\alpha$ is independent of ras mutations in lung adenocarcinoma. Lung Cancer. 2000;29(2):151-157.

57. Volante M, et al. Epidermal growth factor ligand/ receptor loop and downstream signaling activation pattern in completely resected nonsmall cell lung cancer. Cancer. 2007;110(6):1321-1328.

58. Chakraborty S, et al. Constitutive and ligandinduced EGFR signalling triggers distinct and mutually exclusive downstream signalling networks. Nat Commun. 2014;5:5811.

59. Guo G, Gong K, Wohlfeld B, Hatanpaa KJ, Zhao D, Habib AA. Ligand-independent EGFR signaling. Cancer Res. 2015;75(17):3436-3441.

60. Endres NF, et al. Conformational coupling across the plasma membrane in activation of the EGF receptor. Cell. 2013;152(3):543-556.

61. Warta R, Herold-Mende C. Helping EGFR inhibition to block cancer. Nat Neurosci. 2017;20(8):1035-1037.

62. Gullick WJ. Prevalence of aberrant expression of the epidermal growth factor receptor in human cancers. Br Med Bull. 1991;47(1):87-98.

63. Ciardiello F, Tortora G. EGFR antagonists in cancer treatment. N EnglJMed. 2008;358(11):1160-1174. 